\title{
GLOBAL ATTRACTOR FOR THE TIME DISCRETIZED MODIFIED THREE-DIMENSIONAL BÉNARD SYSTEMS
}

\author{
CHAOSHENG ZHU
}

Received 12 December, 2019

\begin{abstract}
In this paper, we aim to study the existence of global attractors for the time discretized modified three-dimensional (3D) Bénard systems. Using the backward implicit Euler scheme, we obtain the time discretization systems of 3D Bénard systems. Then, by the Galerkin method and the Brouwer fixed point theorem, we prove the existence of the solution to this time-discretized systems. On this basis, we proved the existence of the attractor by the compact embedding theorem of Sobolev. Finally, we discuss the limiting behavior of the solution as $N$ tends to infinity.
\end{abstract}

2010 Mathematics Subject Classification: 35B41; 35B45; 35K15

Keywords: Bénard system, global attractor, discretization

\section{INTRODUCTION}

In this work, we study the following 3D Bénard system:

$$
\begin{aligned}
& \left\{\begin{array}{l}
\frac{\partial u}{\partial t}-v \Delta u+F_{N}(\|\nabla u\|)(u \cdot \nabla) u+\xi \omega=f(x)-\nabla p, \\
\operatorname{div} u=0 \\
\left.u\right|_{\partial \Omega}=0,
\end{array}\right. \\
& \left\{\begin{array}{l}
\frac{\partial \omega}{\partial t}-\Delta \omega+(u \cdot \nabla) \omega=g(x), \\
\left.\omega\right|_{\partial \Omega}=0,
\end{array}\right.
\end{aligned}
$$

where $\Omega \subset \mathbb{R}^{3}$ be a bounded smooth domain; $u=u(t, x), \omega=\omega(t, x)$ and $p=p(t, x)$ denote velocity, temperature and pressure of the fluid, respectively; $\nu>0, \xi \in \mathbb{R}^{3}$ are constants; $f: \Omega \rightarrow \mathbb{R}^{3}, g: \Omega \rightarrow \mathbb{R}$ are given functions, and for $N \geq 1, F_{N}(r)=\min \left\{1, \frac{N}{r}\right\}$.

It is well-known that the Bénard system is a dynamic model describing the rate, pressure and temperature of incompressible fluids that are coupled by Navier-Stokes

This work was supported by the National Natural Sciences Foundation of China (No. 11571283). 
equations and convection diffusion equations. This problem is fundamentally important and of both theoretical and practical interest. In recent years, many important achievements have been made in the study of the Bénard system, of which the study of the solution and attractor of the Bénard system is a very important part. For example, in [10], the authors proved the existence of global solution of the equation (1.1)-(1.2) on channel-like domains by the Galerkin method, then they constructed the global $\varphi$ attractor of this systems. To date, many studies have investigated the case of $F_{N}=1$ in the equation (1.1)-(1.2), for example, see [8,9,11,12]. In [8], authors introduced a class of functions which are strongly continuous with respect to the second component of the vector. Then they prove the existence of solutions for the 3D Bénard system, and construct a multi-valued semi-flow generated by such solutions. Moreover, they obtain the existence of a global $\varphi$ attractor for the weak-strong topology. In [12], authors investigate the regularized 3D Bénard problem. Using the averaging technique which will give us the properties of the mean characteristics of the flow, they prove that the global existence and uniqueness of the solutions, and then obtain the existence of the global attractor. In [9], authors study the asymptotic behaviour of weak solutions for the 3D Bénard problem. They first show some regularity properties of the weak solutions of this systems. Then they construct a one parameter family of multi-valued semi-flow and obtain the existence of a global attractor with respect to the weak topology of the phase space. In [11], authors first establish an energy inequality in the space $L^{4}$ for a broader class of weak solutions. Using this inequality, they prove the existence and connectedness of a global attractor in the space $H_{w} \times L^{2}$ for the corresponding $m$-semi-flow.

It is well-known that the discretization method is the basic method to solve the problems of continuum mechanics, which is a method to approximate the physical quantities in continuum mechanics with finite parameters. The laws of continuum mechanics are generally described by differential equations and integral equations. The discretization method approximates the original problem by transforming it into an algebraic equation with finite parameters. The discretization of differential equation mainly refers to the discretization of time and space. The usual discretization methods include finite difference method, finite element method, weighted residual method and so on (see [1, 2, 4, 6, 7, 14, 16]). In [5], the modified 3D Navier-Stokes equations were discretized on the time by finite difference method, then the existence of the global attractor was proved. In the literature [15], the Benjamin-Bona-Mahony equation was discretized on the time by the Crank-Nicolson scheme. Then, using the Galerkin method and the Brouwer fixed point theorem, authors proved that the existence of the solution to this time discretized system. Furthermore, authors showed that the existence of attractor by Sobolev's compact embedding theorem.

The main purpose of this paper is to investigate the long time dynamical behavior of the solution of the discretized, modified 3D Bénard system (1.1)-(1.2) by the idea in $[5,15]$. 
Let us firstly introduce some notations. Set

$$
\begin{aligned}
& \mathbb{H}=\left\{u \in\left(L^{2}(\Omega)\right)^{3}, \operatorname{div} u=0, u \cdot n=0 \text { on } \partial \Omega\right\}, \\
& \mathbb{V}=\left\{u \in\left(H_{0}^{1}(\Omega)\right)^{3}, \operatorname{div} u=0\right\},
\end{aligned}
$$

with norms $\|\cdot\|,\||\cdot|\|$ and scalar products $(\cdot, \cdot),((\cdot, \cdot))$ (the same notations for norms and scalar products also apply to $\left.L^{2}(\Omega), H_{0}^{1}(\Omega)\right)$, where, $n$ is the unit outward normal on $\partial \Omega$. Let

$$
b(u, v, z)=\int_{\Omega} \sum_{i, j=1}^{3} u_{i} \frac{\partial v_{j}}{\partial x_{i}} z_{j} d x, \quad c(u, \omega, \eta)=\int_{\Omega} \sum_{i=1}^{3} u_{i} \frac{\partial \omega}{\partial x_{i}} \eta d x
$$

and $b_{N}(u, v, z)=F_{N}(\|\nabla v\|) b(u, v, z)$. Thanks to Poincaré inequality, we can put

$$
((u, v))=(\nabla u, \nabla v), \quad \quad\|u\|\|=\| \nabla u \| .
$$

We denote by $\mathcal{P}$ the Leray projection of $\left.L^{2}(\Omega)\right)^{d}$ onto $\mathbb{H}$ and by $\mathcal{T}$ the Leray projection of $\left.L^{2}(\Omega)\right)^{d}$ onto $L^{2}(\Omega)$. And we denote by $D\left(A_{1}\right)$ the domain of the Stokes operator $A_{1}=-\mathcal{P} \Delta$ in $\mathbb{H}$, and by $D\left(A_{2}\right)$ the domain of $A_{2}=-\mathcal{T} \Delta$ in $L^{2}(\Omega)$. Obviously, $A_{1}: \mathbb{V} \rightarrow \mathbb{V}^{*}, A_{2}: H_{0}^{1}(\Omega) \rightarrow H^{-1}(\Omega)$ are linear continuous operators and such that

$$
\left\langle A_{1} u, v\right\rangle_{\mathbb{V}, \mathbb{V}^{*}}=(\nabla u, \nabla v), \quad\left\langle A_{2} \omega, \eta\right\rangle_{H_{0}^{1}(\Omega), H^{-1}(\Omega)}=(\nabla \omega, \nabla \eta),
$$

where, $u, v \in \mathbb{V}, \omega, \eta \in H_{0}^{1}(\Omega)$. From the regularity theory for the Stokes equation, it is proved in [13] that $D\left(A_{1}\right)=H^{2}(\Omega)^{3} \cap \mathbb{V}, D\left(A_{2}\right)=H^{2}(\Omega) \cap H_{0}^{1}(\Omega)$, and the following holds true

$$
D\left(A_{1}\right) \subset \mathbb{V} \subset \mathbb{H}, \quad D\left(A_{2}\right) \subset H_{0}^{1}(\Omega) \subset L^{2}(\Omega) .
$$

Therefore,

$$
\begin{array}{cl}
\|\| u\left\|\leq \frac{1}{\sqrt{\lambda}_{1}}\right\| A_{1} u \|, \quad \forall u \in D\left(A_{1}\right), & \|\omega\| \leq \frac{1}{\sqrt{\lambda}_{2}}\left\|A_{2} \omega\right\|, \quad \forall \omega \in D\left(A_{2}\right), \\
\|u\| \leq \frac{1}{\sqrt{\lambda}_{1}}\|u\|, \quad \forall u \in \mathbb{V}, & \|\omega\| \leq \frac{1}{\sqrt{\lambda}_{2}}\|\omega\|, \quad \forall \omega \in H_{0}^{1}(\Omega),
\end{array}
$$

where, $\lambda_{1}>0, \lambda_{2}>0$ are the first eigenvalues of the Stokes operator $A_{1}, A_{2}$, respectively.

We introduce two bilinear operators $B: \mathbb{V} \times \mathbb{V} \rightarrow \mathbb{V}^{*}$ and $C: \mathbb{V} \times H_{0}^{1}(\Omega) \rightarrow H^{-1}(\Omega)$, defined as:

$$
\langle B(u, v), z\rangle_{\mathbb{V}, \mathbb{V}^{*}}=b(u, v, z), \quad\langle C(u, \omega), \eta\rangle_{H_{0}^{1}(\Omega), H^{-1}(\Omega)}=c(u, \omega, \eta),
$$


where, $u, v, z \in \mathbb{V}, \omega, \eta \in H_{0}^{1}(\Omega)$. From [5],

$$
\begin{cases}|b(u, v, z)| \leq C_{b}\|u\|^{\frac{1}{4}}\|u\|^{\frac{3}{4}}\|v\|\|\| z\left\|^{\frac{1}{4}}\right\| z \|^{\frac{3}{4}}, & \forall u, v, z \in \mathbb{V}, \\ |b(u, v, z)| \leq C_{b}\|u\|^{\frac{1}{2}}\left\|A_{1} u\right\|^{\frac{1}{2}}\|\mid\| v\|\| z \|, & \forall u \in D\left(A_{1}\right), v \in \mathbb{V}, z \in \mathbb{H}, \\ |b(u, v, z)| \leq C_{b}\|u\|^{\frac{1}{4}}\left\|A_{1} u\right\|^{\frac{3}{4}}\|v\|\|\| z \|, & \forall u \in D\left(A_{1}\right), v \in \mathbb{V}, z \in \mathbb{H}, \\ b(u, v, v)=0, & \forall u, v \in \mathbb{V} .\end{cases}
$$

Therefore,

$$
b_{N}(u, v, v)=0, \forall u, v \in \mathbb{V} \quad \text { and } \quad\left\langle B_{N}(u, v), z\right\rangle_{\mathbb{V}, \mathbb{V}^{*}}=b_{N}(u, v, z), \forall u, v, z \in \mathbb{V} .
$$

Since $\Omega \subset \mathbb{R}^{3}$ is bounded, there exists a constant $c>0$, which is only related to $\Omega$, such that for all $v \in H^{1}(\Omega)[5]$,

$$
\|v\|_{L^{3}(\Omega)} \leq c\|v\|^{1 / 2}\|v\|^{1 / 2}, \quad \quad\|v\|_{L^{6}(\Omega)} \leq c\|v\| \| .
$$

For $M, N, p, q \in \mathbb{R}_{+}$, there holds [5]

$$
\left|F_{N}(p)-F_{N}(q)\right| \leq \frac{|p-q|}{q}, \quad\left|F_{M}(p)-F_{N}(q)\right| \leq \frac{|M-N|}{q}+\frac{|p-q|}{q} .
$$

By the notations above, the equations (1.1)-(1.2) can be rewritten in the weak form as

$$
\left\{\begin{array}{l}
u_{t}+v A_{1} u+B_{N}(u, u)+\xi \omega=f(x), \\
\omega_{t}+A_{2} \omega+C(u, \omega)=g(x) .
\end{array}\right.
$$

In this paper, we aim to study the existence of global attractors for the time discretized modified three-dimensional (3D) Bénard systems (1.1)-(1.2). To this end, using the backward implicit Euler scheme, we obtain the time discretization systems of (1.6):

$$
\left\{\begin{array}{l}
\frac{u^{m}-u^{m-1}}{k}+v A_{1} u^{m}+B_{N}\left(u^{m}, u^{m}\right)+\xi \omega^{m}=f, \\
\frac{\omega^{m}-\omega^{m-1}}{k}+A_{2} \omega^{m}+C\left(u^{m}, \omega^{m}\right)=g
\end{array}\right.
$$

where $k$ is the time step, and $u^{m} \sim u\left(t^{m}\right), \omega^{m} \sim \omega\left(t^{m}\right)$.

The main results of this paper are as follows. Firstly, by the Galerkin method and the Brouwer fixed point theorem, we prove the existence of the solution to this time-discretized systems (1.7)-(1.8).

Theorem 1. Supposing that $u_{0} \in D\left(A_{1}\right), \omega_{0} \in D\left(A_{2}\right)$. Let $f \in L^{2}(\Omega)^{3}, g \in L^{2}(\Omega)$ be given functions, and let $k>0$. Then there is at least one set of solutions $\left\{u^{m}, \omega^{m}\right\} \in D\left(A_{1}\right) \times D\left(A_{2}\right)$ to (1.7)-(1.8) for $m \geq 1$ be integers.

On this basis, by the compact embedding theorem of Sobolev, we proved the existence of the attractor. 
Theorem 2. Supposing that $u_{0} \in \mathbb{V}$, $\omega_{0} \in H_{0}^{1}(\Omega)$. Let $f \in L^{2}(\Omega)^{3}, g \in L^{2}(\Omega)$ be given functions and let $k>0$ small enough. Then the $C^{0}$ semigroup $S^{m}$ defined by the systems (1.7)-(1.8) has global attractors $\mathcal{A}$ in $\mathbb{V} \times H_{0}^{1}(\Omega)$.

Finally, we discuss the limiting behavior of the solution to (1.7)-(1.8) as $N$ tends to infinity.

Theorem 3. Supposing that $u_{0} \in D\left(A_{1}\right), \omega_{0} \in D\left(A_{2}\right)$. Let $f \in L^{2}(\Omega)^{3}, g \in L^{2}(\Omega)$ be given functions, and let $k>0$. Then, for $m>1$ be integers, the solution sequence $\left\{u_{N}^{m}, \omega_{N}^{m}\right\}_{N}$ of (1.7)-(1.8) converges to the weak solution of the following equations when $N \rightarrow \infty$,

$$
\left\{\begin{array}{l}
\frac{u^{m}-u^{m-1}}{k}+v A_{1} u^{m}+B\left(u^{m}, u^{m}\right)+\xi \omega^{m}=f, \\
\frac{\omega^{m}-\omega^{m-1}}{k}+A_{2} \omega^{m}+C\left(u^{m}, \omega^{m}\right)=g .
\end{array}\right.
$$

This paper is organized as follows. Section 2 proves the existence of solutions and completes the proof of Theorem 1. Section 3 proves the boundedness of solution in phase space. Section 4 proves the continuous dependence of solution on initial value and parameter $N$, and establishes a discrete semigroup $S^{m}$ to complete the proof of Theorem 2. Section 5 discusses the limit behavior of $\left\{u_{N}^{m}, \omega_{N}^{m}\right\}$ as $N$ tends to infinity and completes the proof of Theorem 3 .

\section{EXISTENCE OF SOLUTIONS}

In this section, we construct a weak solution of (1.7)-(1.8) by the Faedo-Galerkin method and the following Brouwer fixed point principle (see [3], 24-29).

Lemma 1 ([3]). Let $X$ be a finite-dimensional space endowed with a scalar product $[\cdot, \cdot]$ and consider a continuous mapping $F: X \rightarrow X$. Suppose that there exists $R_{0}>0$ such that $\left[F\left(U_{0}\right), U_{0}\right]>0$ for all $U_{0} \in X$ with $\left[U_{0}, U_{0}\right]=R_{0}^{2}$. Then there exists $U$ with $[U, U] \leq R_{0}^{2}$ such that $F(U)=0$.

To prove the existence of the solution for (1.7)-(1.8), the following three steps are required:

Step 1: Construct an approximate solution. Let $p \geq 1$ be an integer. For $u^{1}, \cdots, u^{m-1}, \omega^{1}, \cdots, \omega^{m-1}$, we can define the approximate solutions of (1.7)-(1.8) by $u_{p}^{m}=\sum_{i=1}^{p} g_{i p}{ }^{m} e_{i}$ and $\omega_{p}^{m}=\sum_{i=1}^{p} h_{i p}{ }^{m} \bar{e}_{i}$ :

$$
\left\{\begin{array}{l}
\frac{u_{p}^{m}-u^{m-1}}{k}+v A_{1} u_{p}^{m}+B_{N}\left(u_{p}^{m}, u_{p}^{m}\right)+\xi \omega_{p}^{m}=f, \\
\frac{\omega_{p}^{m}-\omega^{m-1}}{k}+A_{2} \omega_{p}^{m}+C\left(u_{p}^{m}, \omega_{p}^{m}\right)=g
\end{array}\right.
$$


where $g_{i p}{ }^{m} \in \mathbb{R},\left\{e_{i}\right\}_{i=1}^{\infty} \subset D\left(A_{1}\right)$, corresponding to the eigenvectors of the operator $A_{1}$, which are ortho-normal base in $\mathbb{H}$ and orthogonal in $\mathbb{V}$; and $h_{i p}{ }^{m} \in \mathbb{R}$, $\left\{\bar{e}_{i}\right\}_{i=1}^{\infty} \subset D\left(A_{2}\right)$, corresponding to the eigenvectors of the operator $A_{2}$, which are ortho-normal base in $L^{2}(\Omega)$ and orthogonal in $H_{0}^{1}(\Omega)$. Let $K_{p}=\left\langle e_{1}, e_{2}, \cdots, e_{p}\right\rangle$ is the space generated by $e_{1}, e_{2}, \cdots, e_{p}$ and $M_{p}=\left\langle\bar{e}_{1}, \bar{e}_{2}, \cdots, \bar{e}_{p}\right\rangle$ is the space generated by $\bar{e}_{1}, \bar{e}_{2}, \cdots, \bar{e}_{p}$, we define operator $Q_{1}: K_{p} \rightarrow K_{p}$ and $Q_{2}: M_{p} \rightarrow M_{p}$ satisfy

$$
\begin{aligned}
& \left(\left(Q_{1}(u), v_{1}\right)\right)=\left(u, v_{1}\right)+v k\left(\nabla u, \nabla v_{1}\right)+k b_{N}\left(u, u, v_{1}\right)+k\left(\xi \omega, v_{1}\right)-\left(u^{m-1}, v_{1}\right)-k\left(f, v_{1}\right) ; \\
& \left(\left(Q_{2}(\omega), v_{2}\right)\right)=\left(\omega, v_{2}\right)+k\left(\nabla \omega, \nabla v_{2}\right)+k c\left(u, \omega, v_{2}\right)-\left(\omega^{m-1}, v_{2}\right)-k\left(g, v_{2}\right) .
\end{aligned}
$$

To apply Lemma 1, we introduce the operator $F(u, \omega)=\left(Q_{1}(u), Q_{2}(\omega)\right)^{\top}$ :

$$
\begin{aligned}
\left(F(u, \omega),\left(v_{1}, v_{2}\right)^{\top}\right) & =\left(\left(Q_{1}(u), Q_{2}(\omega)\right)^{\top},\left(v_{1}, v_{2}\right)^{\top}\right) \\
& =\left(\left(Q_{1}(u), v_{1}\right)\right)+\left(\left(Q_{2}(\omega), v_{2}\right)\right) .
\end{aligned}
$$

Now we need to prove that $F(u, \omega)$ is continuous in $\mathbb{V}$. To this end, let $u_{1}, u_{2}, v_{1} \in K_{p}$ and $\omega_{1}, \omega_{2}, v_{2} \in M_{p}$, we have

$$
\begin{aligned}
( & \left.F\left(u_{1}, \omega_{1}\right)-F\left(u_{2}, \omega_{2}\right),\left(v_{1}, v_{2}\right)^{\top}\right) \\
= & \left(\left(Q_{1}\left(u_{1}\right), Q_{2}\left(\omega_{1}\right)\right)^{\top},\left(v_{1}, v_{2}\right)^{\top}\right)+\left(\left(Q_{1}\left(u_{2}\right), Q_{2}\left(\omega_{2}\right)\right)^{\top},\left(v_{1}, v_{2}\right)^{\top}\right) \\
= & \left(u_{1}, v_{1}\right)+v k\left(\nabla u_{1}, \nabla v_{1}\right)+k b_{N}\left(u_{1}, u_{1}, v_{1}\right)+k\left(\xi \omega_{1}, v_{1}\right)-\left(u^{m-1}, v_{1}\right) \\
& -k\left(f, v_{1}\right)+\left(\omega_{1}, v_{2}\right)+k\left(\nabla \omega_{1}, \nabla v_{2}\right)+k c\left(u_{1}, \omega_{1}, v_{2}\right)-\left(\omega^{m-1}, v_{2}\right)-k\left(g, v_{2}\right) \\
& -\left[\left(u_{2}, v_{1}\right)+v k\left(\nabla u_{2}, \nabla v_{1}\right)+k b_{N}\left(u_{2}, u_{2}, v_{1}\right)+k\left(\xi \omega_{2}, v_{1}\right)-\left(u^{m-1}, v_{1}\right)\right. \\
& \left.-k\left(f, v_{1}\right)+\left(\omega_{2}, v_{2}\right)+k\left(\nabla \omega_{2}, \nabla v_{2}\right)+k c\left(u_{2}, \omega_{2}, v_{2}\right)-\left(\omega^{m-1}, v_{2}\right)-k\left(g, v_{2}\right)\right] \\
= & \left(u_{1}-u_{2}, v_{1}\right)+v k\left(\nabla\left(u_{1}-u_{2}\right), \nabla v_{1}\right)+k\left(\xi\left(\omega_{1}-\omega_{2}\right), v_{1}\right) \\
& +k\left(\nabla\left(\omega_{1}-\omega_{2}\right), \nabla v_{2}\right)+\left(\omega_{1}-\omega_{2}, v_{2}\right) \\
& +k\left[b_{N}\left(u_{1}, u_{1}, v_{1}\right)-b_{N}\left(u_{2}, u_{2}, v_{1}\right)\right]+k\left[c\left(u_{1}, \omega_{1}, v_{2}\right)-c\left(u_{2}, \omega_{2}, v_{2}\right)\right] .
\end{aligned}
$$

Here, by using Poincaré inequality, we can get

$$
\begin{aligned}
& \left(u_{1}-u_{2}, v_{1}\right)+v k\left(\nabla\left(u_{1}-u_{2}\right), \nabla v_{1}\right)+k\left(\xi\left(\omega_{1}-\omega_{2}\right), v_{1}\right) \\
& +k\left(\nabla\left(\omega_{1}-\omega_{2}\right), \nabla v_{2}\right)+\left(\omega_{1}-\omega_{2}, v_{2}\right) \\
& \leq\left[C\left\|v_{1}\right\|+v k\left\|v_{1}\right\| \mid \|\right]\left\|u_{1}-u_{2}\right\| \mid+\left[C\left\|v_{2}\right\|+k C|\xi|\left\|v_{1}\right\|+k\left\|v_{2}\right\| \mid \|\right]\left\|\omega_{1}-\omega_{2}\right\| .
\end{aligned}
$$

And by the definition of $F_{N}$ and (1.3), we obtain

$$
\begin{aligned}
& b_{N}\left(u_{1}, u_{1}, v_{1}\right)-b_{N}\left(u_{2}, u_{2}, v_{1}\right)=F_{N}\left(\left\|u_{1} \mid\right\|\right) b\left(u_{1}, u_{1}, v_{1}\right)-F_{N}\left(\left\|\left|u_{2} \|\right|\right) b\left(u_{2}, u_{2}, v_{1}\right)\right. \\
& =F_{N}\left(\left\|u_{1}\right\|||\right) b\left(u_{1}-u_{2}, u_{1}, v_{1}\right)+F_{N}\left(\left\|\left|u_{2} \|\right| \mid\right) b\left(u_{2}, u_{1}-u_{2}, v_{1}\right)\right.
\end{aligned}
$$




$$
\begin{aligned}
& +\left[F_{N}\left(\left\|u_{1}\right\| \mid\right)-F_{N}\left(\left\|u_{2}\right\|||\right)\right] b\left(u_{2}, u_{1}, v_{1}\right) \\
& \leq C N|| u_{1}-u_{2}||||\left|v_{1}\right|||+C||\left|u_{1}-u_{2}\right||||| u_{1}|||||| v_{1} \mid \| \\
& \leq\left[C N\left|\left\|v_{1}\right\|\right|+C\left|\left\|u_{1}\left|\left\|\left|\left\|v_{1}\right\|\right|\right]\right|\right\| u_{1}-u_{2} \|\right| .\right.
\end{aligned}
$$

On the other hand, for $c(u, \omega, \eta)$, we have

$$
|c(u, \omega, \eta)| \leq\|u\|_{L^{6}(\Omega)}\|\nabla \omega\|_{L^{2}(\Omega)}\|\eta\|_{L^{3}(\Omega)} \leq C|\|u\||\||\|\omega\||\||\|\eta \mid\|,
$$

and $c(u, \omega, \omega)=0$, so we can get

$$
\begin{aligned}
c\left(u_{1}, \omega_{1}, v_{2}\right)-c\left(u_{2}, \omega_{2}, v_{2}\right) & =c\left(u_{1}-u_{2}, \omega_{1}, v_{2}\right)+c\left(u_{2}, \omega_{1}-\omega_{2}, v_{2}\right) \\
& \leq C\left\|| \omega _ { 1 } | | | | | | v _ { 2 } | | | | | \left|u_{1}-u_{2}\left\|||+C\left|\left\|u_{2}|||||| v_{2}\right\|\right||| \mid \omega_{1}-\omega_{2}\right\| .\right.\right.
\end{aligned}
$$

Thus, by (2.3)-(2.6), we obtain

$$
\begin{aligned}
& \left(F\left(u_{1}, \omega_{1}\right)-F\left(u_{2}, \omega_{2}\right),\left(v_{1}, v_{2}\right)^{\top}\right) \\
& \leq\left[C\left\|v_{1}\right\|+v k\left\|\left|v_{1}\left\|\left|+C N k\left\|v_{1}\right\|\right|+C k\left|\left\|u_{1}\right\|\right|\right\| v_{1}\||+C|\| \omega_{1}\left\|\left|\left\|\left|v_{2} \|\right|\right]\right|\right\| u_{1}-u_{2}\|\|\right.\right.\right. \\
& +\left[C\left\|v_{2}\right\|+k C|\xi|\left\|v_{1}\right\|+k\left|\left\|v _ { 2 } \left|\left\|+C k\left|\left\|u _ { 2 } \left|\left\|| \| v _ { 2 } | \| ] \left|\left\|\omega_{1}-\omega_{2} \mid\right\| .\right.\right.\right.\right.\right.\right.\right.\right.\right.\right.
\end{aligned}
$$

It is easy to know that $F(u, \omega)$ is continuous in $\mathbb{V}$. Next, let $k$ be small enough such that $1-\frac{k}{2}>0$ and $1-\frac{k}{2}|\xi|^{2}>0$. For $\{u, \omega\} \in K_{p} \times M_{p}$, by Cauchy-Schwarz and Poincaré inequality, we find

$$
\begin{aligned}
& \left(F(u, \omega),(u, \omega)^{\top}\right)=\left(\left(Q_{1}(u), Q_{2}(\omega)\right)^{\top},(u, \omega)^{\top}\right) \\
& =(u, u)+v k(\nabla u, \nabla u)+k b_{N}(u, u, u)+k(\xi \omega, u)-\left(u^{m-1}, u\right)-k(f, u) \\
& \quad+(\omega, \omega)+k(\nabla \omega, \nabla \omega)+k c(u, \omega, \omega)-\left(\omega^{m-1}, \omega\right)-k(g, \omega) \\
& =\|u\|^{2}+v k\|u\| \|^{2}+k(\xi \omega, u)-\left(u^{m-1}, u\right)-k(f, u) \\
& \quad+\|\omega\|^{2}+k\|\omega\|^{2}-\left(\omega^{m-1}, \omega\right)-k(g, \omega) \\
& \geq\|u\|^{2}+v k\|u\|\left\|^{2}+\right\| \omega\left\|^{2}+k\right\| \omega\|\|^{2}-k\|\xi \omega\|\|u\| \\
& \quad-\left\|u^{m-1}\right\|\|u\|-k\|f\|\|u\|-\left\|\omega^{m-1}\right\|\|\omega\|-k\|g\|\|\omega\| \\
& \geq\|u\|^{2}+v k\|u\|\left\|^{2}+\right\| \omega\left\|^{2}+k\right\| \omega\|\|^{2}-\frac{k}{2}\left[\|\xi \omega\|^{2}+\|u\|^{2}\right] \\
& \quad-\frac{\left\|u^{m-1}\right\|}{\sqrt{\lambda_{1}}}\|u\|\left\|-k \frac{\|f\|}{\sqrt{\lambda_{1}}}\right\| u\left\|-\frac{\left\|\omega^{m-1}\right\|}{\sqrt{\lambda_{2}}}\right\| \omega\left\|-k \frac{\|g\|}{\sqrt{\lambda_{2}}}\right\| \omega\|\| \\
& =\left[1-\frac{k}{2}\right]\|u\|^{2}+v k\|u\|^{2}+\left[1-\frac{k}{2}|\xi|^{2}\right]\|\omega\|^{2}+k\|\omega\| \|^{2}
\end{aligned}
$$




$$
\begin{aligned}
& -\frac{\left\|u^{m-1}\right\|}{\sqrt{\lambda_{1}}}\|u\|-k \frac{\|f\|}{\sqrt{\lambda_{1}}}\|u\|-\frac{\left\|\omega^{m-1}\right\|}{\sqrt{\lambda_{2}}}\|\omega\| \mid-k \frac{\|g\|}{\sqrt{\lambda_{2}}}\|\omega\| \\
\geq & v k\|u\|\left\|^{2}+k\right\| \omega\left\|^{2}-\frac{\left\|u^{m-1}\right\|}{\sqrt{\lambda_{1}}}\right\| u\left\|-k \frac{\|f\|}{\sqrt{\lambda_{1}}}\right\| u\left\|-\frac{\left\|\omega^{m-1}\right\|}{\sqrt{\lambda_{2}}}\right\| \omega\left\|-k \frac{\|g\|}{\sqrt{\lambda_{2}}}\right\| \omega\|\| \\
= & \|u\|\left[v k\|\| u \|-\frac{\left\|u^{m-1}\right\|}{\sqrt{\lambda_{1}}}-k \frac{\|f\|}{\sqrt{\lambda_{1}}}\right]+\|\omega\|\left[k\|\omega\|-\frac{\left\|\omega^{m-1}\right\|}{\sqrt{\lambda_{2}}}-k \frac{\|g\|}{\sqrt{\lambda_{2}}}\right] .
\end{aligned}
$$

Let $r_{1}>\frac{\left\|u^{m-1}\right\|+k\|f\|}{v k \sqrt{\lambda_{1}}}$ and $r_{2}>\frac{\left\|\omega^{m-1}\right\|+k\|g\|}{k \sqrt{\lambda_{2}}}$, for any $\{u, \omega\} \in K_{p} \times M_{p}$ with $\|u\|=r_{1}$ and $\|\omega\|=r_{2}$, one has $\left(F(u, \omega),(u, \omega)^{\top}\right)>0$. Thus, from Lemma 1, we can find $\left(u^{*}, \omega^{*}\right)$ satisfy $F\left(u^{*}, \omega^{*}\right)=0$, which is $\left(Q_{1}\left(u^{*}\right), Q_{2}\left(\omega^{*}\right)\right)^{\top}=\mathbf{0}$, and so $\left\{\begin{array}{l}Q_{1}\left(u^{*}\right)=0, \\ Q_{2}\left(\omega^{*}\right)=0 .\end{array}\right.$

Therefore, the approximate solution $\left\{u_{p}^{m}, \omega_{p}^{m}\right\}$ exists.

Step 2: Some priori estimates. For $k$ and $m$ are fixed, we want to get a priori estimates independent of $p$. Multiplying the equation (2.1) by $u_{p}^{m}$ and the equation (2.2) by $\omega_{p}^{m}$, we obtain

$$
\begin{aligned}
\left\|u_{p}^{m}\right\|^{2}+\left\|\omega_{p}^{m}\right\|^{2}+\left\|u_{p}^{m}-u^{m-1}\right\|^{2}+\left\|\omega_{p}^{m}-\omega^{m-1}\right\|^{2}+2 v k\left\|u_{p}^{m}\right\|^{2}+2 k\left\|\omega_{p}^{m}\right\|^{2} \\
=2 k\left(f, u_{p}^{m}\right)+2 k\left(g, \omega_{p}^{m}\right)+\left\|u^{m-1}\right\|^{2}+\left\|\omega^{m-1}\right\|^{2}-2 k\left(\xi \omega_{p}^{m}, u_{p}^{m}\right) \\
\leq 2 k\|f\|\left\|u_{p}^{m}\right\|+2 k\|g\|\left\|\omega_{p}^{m}\right\|+2 k|\xi|\left\|\omega_{p}^{m}\right\|\left\|u_{p}^{m}\right\|+\left\|u^{m-1}\right\|^{2}+\left\|\omega^{m-1}\right\|^{2} \\
\leq \frac{k}{v \lambda_{1}}\|f\|^{2}+v k\|\| u_{p}^{m}\left\|^{2}+\frac{k}{\lambda_{2}}\right\| g\left\|^{2}+k\right\| \omega_{p}^{m}\left\|^{2}+k|\xi|\right\| \omega_{p}^{m} \|^{2} \\
\quad+k|\xi|\left\|u_{p}^{m}\right\|^{2}+\left\|u^{m-1}\right\|^{2}+\left\|\omega^{m-1}\right\|^{2} .
\end{aligned}
$$

Therefore,

$$
\begin{aligned}
& (1-k|\xi|)\left\|u_{p}^{m}\right\|^{2}+(1-k|\xi|)\left\|\omega_{p}^{m}\right\|^{2} \\
& \quad+\left\|u_{p}^{m}-u^{m-1}\right\|^{2}+\left\|\omega_{p}^{m}-\omega^{m-1}\right\|^{2}+v k\|\| u_{p}^{m}\left\|^{2}+k\right\| \omega_{p}^{m} \|^{2} \\
& \leq \frac{k}{v \lambda_{1}}\|f\|^{2}+\frac{k}{\lambda_{2}}\|g\|^{2}+\left\|u^{m-1}\right\|^{2}+\left\|\omega^{m-1}\right\|^{2} .
\end{aligned}
$$

Let $k$ be small enough such that $1-k|\xi|>0$, one has

$$
\begin{aligned}
& \left\|u_{p}^{m}\right\|^{2}+\left\|\omega_{p}^{m}\right\|^{2}+\frac{1}{1-k|\xi|}\left\|u_{p}^{m}-u^{m-1}\right\|^{2} \\
& \quad+\frac{1}{1-k|\xi|}\left\|\omega_{p}^{m}-\omega^{m-1}\right\|^{2}+\frac{v k}{1-k|\xi|}\left\|u_{p}^{m}\right\|^{2}+\frac{k}{1-k|\xi|}\left\|\omega_{p}^{m}\right\|^{2} \\
& \leq \frac{1}{1-k|\xi|}\left[\frac{k}{v \lambda_{1}}\|f\|^{2}+\frac{k}{\lambda_{2}}\|g\|^{2}+\left\|u^{m-1}\right\|^{2}+\left\|\omega^{m-1}\right\|^{2}\right] .
\end{aligned}
$$


Now taking the $L^{2}$ inner product of the equation (2.1) with $A_{1} u_{p}^{m}$ and of the equation (2.2) with $A_{2} \omega_{p}^{m}$, we obtain

$$
\begin{aligned}
\vee & k\left\|A_{1} u_{p}^{m}\right\|^{2}+k\left\|A_{2} \omega_{p}^{m}\right\|^{2} \\
= & k\left(f, A_{1} u_{p}^{m}\right)+k\left(g, A_{2} \omega_{p}^{m}\right)-\left(u_{p}^{m}-u^{m-1}, A_{1} u_{p}^{m}\right)-\left(\omega_{p}^{m}-\omega^{m-1}, A_{2} \omega_{p}^{m}\right) \\
& -k b_{N}\left(u_{p}^{m}, u_{p}^{m}, A_{1} u_{p}^{m}\right)-k c\left(u_{p}^{m}, \omega_{p}^{m}, A_{2} \omega_{p}^{m}\right)-k\left(\xi \omega_{p}^{m}, A_{1} u_{p}^{m}\right) \\
\leq & k\|f\|\left\|A_{1} u_{p}^{m}\right\|+k\|g\|\left\|A_{2} \omega_{p}^{m}\right\|+\left\|u_{p}^{m}-u^{m-1}\right\|\left\|A_{1} u_{p}^{m}\right\|+\left\|\omega_{p}^{m}-\omega^{m-1}\right\|\left\|A_{2} \omega_{p}^{m}\right\| \\
& +k \frac{N}{\|\| u_{p}^{m}\|\|}\left\|\left(u_{p}^{m} \cdot \nabla\right) u_{p}^{m}\right\|\left\|A_{1} u_{p}^{m}\right\|+k\left|c\left(u_{p}^{m}, \omega_{p}^{m}, A_{2} \omega_{p}^{m}\right)\right|+k\left\|\xi \omega_{p}^{m}\right\|\left\|A_{1} u_{p}^{m}\right\| \\
\leq & k\|f\|\left\|A_{1} u_{p}^{m}\right\|+k\|g\|\left\|A_{2} \omega_{p}^{m}\right\|+\left\|u_{p}^{m}-u^{m-1}\right\|\left\|A_{1} u_{p}^{m}\right\|+\left\|\omega_{p}^{m}-\omega^{m-1}\right\|\left\|A_{2} \omega_{p}^{m}\right\| \\
& +k \frac{N}{\left\|u_{p}^{m}\right\| \mid} C\left\|u_{p}^{m}\right\|\|\| u_{p}^{m}\left\|\left.\right|^{\frac{1}{2}}\right\| A_{1} u_{p}^{m}\left\|^{\frac{3}{2}}+k\left|c\left(u_{p}^{m}, \omega_{p}^{m}, A_{2} \omega_{p}^{m}\right)\right|+k|\xi|\right\| \omega_{p}^{m}\|\| A_{1} u_{p}^{m} \| \\
\leq & k\|f\|\left\|A_{1} u_{p}^{m}\right\|+k\|g\|\left\|A_{2} \omega_{p}^{m}\right\|+\left\|u_{p}^{m}-u^{m-1}\right\|\left\|A_{1} u_{p}^{m}\right\|+\left\|\omega_{p}^{m}-\omega^{m-1}\right\|\left\|A_{2} \omega_{p}^{m}\right\| \\
& +C N k\left\|u_{p}^{m}\right\|\left\|^{\frac{1}{2}}\right\| A_{1} u_{p}^{m}\left\|^{\frac{3}{2}}+k\left|c\left(u_{p}^{m}, \omega_{p}^{m}, A_{2} \omega_{p}^{m}\right)\right|+k|\xi|\right\| \omega_{p}^{m}\|\| A_{1} u_{p}^{m} \| .
\end{aligned}
$$

Since

$\left|c\left(u, \omega, A_{2} \omega\right)\right| \leq\|u\|_{L^{\infty}(\Omega)}\|\nabla \omega\|_{L^{2}(\Omega)}\left\|A_{2} \omega\right\|_{L^{2}(\Omega)} \leq C\left[\frac{\varepsilon}{2}\|u\|\left\|^{2}\right\| \omega\left\|^{2}+\frac{1}{2 \varepsilon}\right\| A_{2} \omega \|^{2}\right]$, we can get

$$
\begin{aligned}
\left\|A_{1} u_{p}^{m}\right\|^{2}+\left\|A_{2} \omega_{p}^{m}\right\|^{2} \leq & C\|f\|^{2}+C\|g\|^{2}+C\left\|u_{p}^{m}-u^{m-1}\right\|^{2}+C\left\|\omega_{p}^{m}-\omega^{m-1}\right\|^{2} \\
& +C N k\|\| u_{p}^{m}\left\|\left.\right|^{2}+c k|\xi|\right\| \omega_{p}^{m}\left\|^{2}+c\left|\left\|u_{p}^{m}\right\|\right|^{2}\right\| \omega_{p}^{m} \|\left.\right|^{2} .
\end{aligned}
$$

By (2.7)-(2.8), we can get

$$
\left\|A_{1} u_{p}^{m}\right\|^{2}+\left\|A_{2} \omega_{p}^{m}\right\|^{2} \leq C\left(\|f\|,\|g\|, \vee, \lambda_{1}, \lambda_{2}, k,\left\|u^{m-1}\right\|,\left\|\omega^{m-1}\right\|, N,|\xi|\right) .
$$

Step 3: Passage to the limit. For $k$ and $m$ fixed, from the above inequality we can see $\left\{u_{p}^{m}\right\}_{p},\left\{\omega_{p}^{m}\right\}_{p}$ are bounded in $D\left(A_{1}\right)$ and $D\left(A_{2}\right)$, respectively. Thus one can extract from $\left\{u_{p}^{m}\right\}_{p}$ and $\left\{\omega_{p}^{m}\right\}_{p}$ subsequences respectively, denoted also by $\left\{u_{p}^{m}\right\}_{p}$, $\left\{\omega_{p}^{m}\right\}_{p}$, such that $u_{p}^{m} \rightarrow u^{m}, p \rightarrow \infty$ in $D\left(A_{1}\right)$, and $\omega_{p}^{m} \rightarrow \omega^{m}, p \rightarrow \infty$ in $D\left(A_{2}\right)$. But, $D\left(A_{1}\right) \hookrightarrow \mathbb{V}$ and $D\left(A_{2}\right) \hookrightarrow H_{0}^{1}(\Omega)$ are compact, so $u_{p}^{m} \rightarrow u^{m}, p \rightarrow \infty$ in $\mathbb{V}$, and $\omega_{p}^{m} \rightarrow$ $\omega^{m}, p \rightarrow \infty$ in $H_{0}^{1}(\Omega)$. Next, we prove that $\left\{u^{m}, \omega^{m}\right\}$ is the solution of (2.1)-(2.2). For the purpose, it is enough to show that

$$
\lim _{p \rightarrow \infty} b_{N}\left(u_{p}^{m}, u_{p}^{m}, v_{1}\right)=b_{N}\left(u^{m}, u^{m}, v_{1}\right) \text { and } \lim _{p \rightarrow \infty} c\left(u_{p}^{m}, \omega_{p}^{m}, v_{2}\right)=c\left(u^{m}, \omega^{m}, v_{2}\right) .
$$

To this end, we calculate as follows

$$
\begin{aligned}
& b_{N}\left(u_{p}^{m}, u_{p}^{m}, v_{1}\right)-b_{N}\left(u^{m}, u^{m}, v_{1}\right)=F_{N}\left(\left\|u_{p}^{m}\right\| \|\right) b\left(u_{p}^{m}, u_{p}^{m}, v_{1}\right)-F_{N}\left(\left\|u^{m}\right\| \|\right) b\left(u^{m}, u^{m}, v_{1}\right) \\
& =F_{N}\left(\left\|u_{p}^{m}\right\| \|\right)\left[b\left(u_{p}^{m}, u_{p}^{m}, v_{1}\right)-b\left(u^{m}, u^{m}, v_{1}\right)\right]
\end{aligned}
$$




$$
\begin{aligned}
& +F_{N}\left(\left\|u_{p}^{m}\right\| \mid\right) b\left(u^{m}, u^{m}, v_{1}\right)-F_{N}\left(\left\|u^{m} \mid\right\|\right) b\left(u^{m}, u^{m}, v_{1}\right) \\
= & F_{N}\left(\left\|u_{p}^{m}\right\|\right)\left[b\left(u_{p}^{m}, u_{p}^{m}, v_{1}\right)-b\left(u^{m}, u^{m}, v_{1}\right)\right]+\left[F_{N}\left(\left\|u_{p}^{m}\right\| \mid\right)-F_{N}\left(\left\|u^{m}\right\|\right)\right] b\left(u^{m}, u^{m}, v_{1}\right) \\
\leq & \left|b\left(u_{p}^{m}, u_{p}^{m}, v_{1}\right)-b\left(u^{m}, u^{m}, v_{1}\right)\right|+\frac{||\left|u_{p}^{m}\right|\left\||-|\left|u^{m}\right|\right\| \mid}{\left\|u^{m}\right\| \mid}\left|b\left(u^{m}, u^{m}, v_{1}\right)\right| .
\end{aligned}
$$

Following [13], one has $\left|b\left(u_{p}^{m}, u_{p}^{m}, v_{1}\right)-b\left(u^{m}, u^{m}, v_{1}\right)\right| \rightarrow 0, p \rightarrow \infty$. And since $\left|b\left(u^{m}, u^{m}, v_{1}\right)\right|$ is bounded uniformly with respect to $p$, one sees that

$$
\frac{||\left|u_{p}^{m}\right||-||| u^{m}|\||}{\left\|\left|u^{m} \|\right|\right.}\left|b\left(u^{m}, u^{m}, v_{1}\right)\right| \rightarrow 0, p \rightarrow \infty .
$$

Therefore, $\lim _{p \rightarrow \infty} b_{N}\left(u_{p}^{m}, u_{p}^{m}, v_{1}\right)=b_{N}\left(u^{m}, u^{m}, v_{1}\right)$. Similarly, we can get

$$
\lim _{p \rightarrow \infty} c\left(u_{p}^{m}, \omega_{p}^{m}, v_{2}\right)=c\left(u^{m}, \omega^{m}, v_{2}\right) .
$$

So $\left\{u^{m}, \omega^{m}\right\}$ is the solution of (1.7)-(1.8). The proof of Theorem 1 is completed.

\section{BOUNDEDNESS}

Let $\left\{u^{m}, \omega^{m}\right\}$ be the solution sequence of (1.7)-(1.8), we are going to show that the boundedness of $\left\{u^{m}, \omega^{m}\right\}$ in $\mathbb{H} \times L^{2}(\Omega), \mathbb{V} \times H_{0}^{1}(\Omega)$ and $D\left(A_{1}\right) \times D\left(A_{2}\right)$ respectively.

\subsection{Boundedness in $\mathbb{H} \times L^{2}(\Omega)$}

Lemma 2. Let $\left\{u^{m}, \omega^{m}\right\}_{m}$ be the solution sequence of (1.7)-(1.8), constructed in Theorem 1. Then for all integers $m \geq 1,\left\{u^{m}, \omega^{m}\right\}$ remain bounded in $\mathbb{H} \times L^{2}(\Omega)$, in the following sense,

$$
\begin{array}{lrl}
\left\|\omega^{m}\right\|^{2} \leq K_{1}, & \forall m \geq 1 ; \\
\left\|u^{m}\right\|^{2} \leq K_{1}^{*}, & \forall m \geq 1 ; \\
\sum_{m=i}^{L-1}\left\|\omega^{m}-\omega^{m-1}\right\|^{2}+\sum_{m=i}^{L-1} k\left\|\omega^{m}\right\|^{2} \leq \frac{k}{\lambda_{2}}\|g\|^{2}(L-i)+K_{1}, & L \geq i ; \\
\sum_{m=i}^{L-1}\left\|u^{m}-u^{m-1}\right\|^{2}+\sum_{m=i}^{L-1} v k\left\|u^{m}\right\|^{2} & \\
\leq K_{1}^{*}+(L-i)\left[\frac{2 k}{v \lambda_{1}}\|f\|^{2}+\frac{2 k|\xi|^{2}}{v \lambda_{1}} K_{1}\right], & L \geq i,
\end{array}
$$

where $K_{1} \triangleq\left\|\omega^{0}\right\|^{2}+\frac{\|g\|^{2}}{\lambda_{2}^{2}}, \quad K_{1}^{*} \triangleq\left\|u^{0}\right\|^{2}+\frac{2\|f\|^{2}}{v^{2} \lambda_{1}^{2}}+\frac{2|\xi|^{2}}{v^{2} \lambda_{1}^{2}} K_{1}$.

Proof. Taking the $L^{2}$ inner product of the equation (1.8) with $2 k \omega^{m}$, we obtain

$$
\left\|\omega^{m}\right\|^{2}+\left\|\omega^{m}-\omega^{m-1}\right\|^{2}+2 k\left\|\omega^{m} \mid\right\|^{2}=2 k\left(g, \omega^{m}\right)+\left\|\omega^{m-1}\right\|^{2}
$$




$$
\leq 2 k \frac{\|g\|}{\sqrt{\lambda_{2}}}\left\|\omega^{m}\right\|+\left\|\omega^{m-1}\right\|^{2} \leq \frac{k}{\lambda_{2}}\|g\|^{2}+k\left\|\omega^{m}\right\|^{2}+\left\|\omega^{m-1}\right\|^{2} .
$$

Thus, we can get

$$
\left\|\omega^{m}\right\|^{2}+\left\|\omega^{m}-\omega^{m-1}\right\|^{2}+k\left\|\omega^{m}\right\|^{2} \leq \frac{k}{\lambda_{2}}\|g\|^{2}+\left\|\omega^{m-1}\right\|^{2} .
$$

By Poincaré inequality, we have

$$
\left\|\omega^{m}\right\|^{2} \leq \frac{1}{1+k \lambda_{2}}\left\|\omega^{m-1}\right\|^{2}+\frac{1}{1+k \lambda_{2}} \frac{k}{\lambda_{2}}\|g\|^{2} .
$$

Using the above inequality recursively, we find

$$
\left\|\omega^{m}\right\|^{2} \leq \frac{1}{\left(1+k \lambda_{2}\right)^{m}}\left\|\omega^{0}\right\|^{2}+\frac{\|g\|^{2}}{\lambda_{2}^{2}}\left[1-\frac{1}{\left(1+k \lambda_{2}\right)^{m}}\right] \leq\left\|\omega^{0}\right\|^{2}+\frac{\|g\|^{2}}{\lambda_{2}^{2}} \triangleq K_{1},
$$

that is, equation (3.1) holds. On the other hand, taking the $L^{2}$ inner product of the equation (1.7) with $2 k u^{m}$, we obtain

$$
\begin{aligned}
\left\|u^{m}\right\|^{2}+\left\|u^{m}-u^{m-1}\right\|^{2}+2 v k\left\|u^{m}\right\|^{2} & =2 k\left(f, u^{m}\right)+\left\|u^{m-1}\right\|^{2}-2 k\left(\xi \omega^{m}, u^{m}\right) \\
& \leq 2 k\|f\|\left\|u^{m}\right\|+\left\|u^{m-1}\right\|^{2}+2 k\left\|\xi \omega^{m}\right\|\left\|u^{m}\right\| \\
& \leq \frac{2 k}{\sqrt{\lambda_{1}}}\|f\|\|\| u^{m}\|+\| u^{m-1}\left\|^{2}+\frac{2 k|\xi|}{\sqrt{\lambda_{1}}}\right\| \omega^{m}\|\| u^{m} \| \\
& \leq \frac{2 k}{v \lambda_{1}}\|f\|^{2}+v k\|\| u^{m}\left\|^{2}+\right\| u^{m-1}\left\|^{2}+\frac{2 k|\xi|^{2}}{v \lambda_{1}}\right\| \omega^{m} \|^{2} .
\end{aligned}
$$

Combination (3.6) imply that

$$
\left\|u^{m}\right\|^{2}+\left\|u^{m}-u^{m-1}\right\|^{2}+v k\|\| u^{m}\left\|^{2} \leq\right\| u^{m-1}\left\|^{2}+\frac{2 k}{v \lambda_{1}}\right\| f \|^{2}+\frac{2 k|\xi|^{2}}{v \lambda_{1}} K_{1} .
$$

Using Poincaré inequality again, we can get

$$
\left(1+v k \lambda_{1}\right)\left\|u^{m}\right\|^{2} \leq\left\|u^{m-1}\right\|^{2}+\frac{2 k}{v \lambda_{1}}\|f\|^{2}+\frac{2 k|\xi|^{2}}{\nu \lambda_{1}} K_{1} .
$$

Using the above inequality recursively, we find

$$
\begin{aligned}
\left\|u^{m}\right\|^{2} & \leq \frac{1}{\left(1+v k \lambda_{1}\right)^{m}}\left\|u^{0}\right\|^{2}+\left[1-\frac{1}{\left(1+v k \lambda_{1}\right)^{m}}\right]\left[\frac{2\|f\|^{2}}{v^{2} \lambda_{1}^{2}}+\frac{2|\xi|^{2}}{v^{2} \lambda_{1}^{2}} K_{1}\right] \\
& \leq\left\|u^{0}\right\|^{2}+\frac{2\|f\|^{2}}{v^{2} \lambda_{1}^{2}}+\frac{2|\xi|^{2}}{v^{2} \lambda_{1}^{2}} K_{1} \triangleq K_{1}^{*},
\end{aligned}
$$

that is, the equation (3.2) holds. Adding up (3.5) with $m$ from $i$ to $L-1$, we find

$$
\left\|\omega^{L-1}\right\|^{2}+\sum_{m=i}^{L-1}\left\|\omega^{m}-\omega^{m-1}\right\|^{2}+\sum_{m=i}^{L-1} k\left\|\omega^{m}\right\|^{2} \leq \frac{k}{\lambda_{2}}\|g\|^{2}(L-i)+\left\|\omega^{i-1}\right\|^{2} .
$$


Combination (3.6) imply that (3.3) holds. Adding up (3.7) with $m$ from $i$ to $L-1$, we find

$$
\left\|u^{L-1}\right\|^{2}+\sum_{m=i}^{L-1}\left\|u^{m}-u^{m-1}\right\|^{2}+\sum_{m=i}^{L-1} v k\left\|u^{m}\right\|^{2} \leq\left\|u^{i-1}\right\|^{2}+(L-i)\left[\frac{2 k}{v \lambda_{1}}\|f\|^{2}+\frac{2 k|\xi|^{2}}{v \lambda_{1}} K_{1}\right] .
$$

Combination (3.8) imply that (3.4) holds. The proof of Lemma 2 is completed.

\subsection{Boundedness in $\mathbb{V} \times H_{0}^{1}(\Omega)$}

Lemma 3 ([5]). Let $\left\{x_{m}\right\},\left\{y_{m}\right\},\left\{z_{m}\right\}$ be non-negative sequences. Assume that there are integers $m_{0}, m_{1}$ such that, for $k>0$,

$$
\begin{aligned}
k y_{m} & <\frac{1}{2}, & \forall m & \geq m_{0}, \\
\left(1-k y_{m}\right) x_{m} & \leq x_{m-1}+k z_{m}, & \forall m & >m_{0}+m_{1} .
\end{aligned}
$$

and that for all integers $m * \geq m_{0}, k \sum_{m=m *}^{m *+m_{1}} y_{m} \leq a_{1}, k \sum_{m=m *}^{m *+m_{1}} z_{m} \leq a_{2}, k \sum_{m=m *}^{m *+m_{1}} x_{m} \leq a_{3}$. Then

$$
x_{m} \leq\left[\frac{a_{3}}{k m_{1}}+a_{2}\right] e^{4 a_{1}}, \quad \forall m>m_{0}+m_{1} .
$$

Lemma 4. Let $\left\{u^{m}, \omega^{m}\right\}_{m}$ be the solutions sequence of (1.7)-(1.8), constructed in Theorem 1. Then for all integers $m \geq 1,\left\{u^{m}, \omega^{m}\right\}$ remain bounded in $\mathbb{V} \times H_{0}^{1}(\Omega)$, in the following sense, there exists positive constants $C, a_{1}, a_{2}, a_{3}$, such that

$$
\begin{array}{lr}
\left.\left\|\omega^{m}\right\|\right|^{2} \leq K_{3}, & \forall m \geq 1 ; \\
\left\|u^{m}\right\| \|^{2} \leq K_{2}, & \forall m \geq 1 ; \\
\sum_{m=i}^{L-1}\left\|u^{m}-u^{m-1}\right\|^{2}+\sum_{m=i}^{L-1} \frac{v k}{16}\left\|A_{1} u^{m}\right\|^{2} & \\
\quad \leq K_{2}+C\left[\frac{\|f\|^{2}}{v}+\frac{N^{8}}{v^{7}} K_{1}^{*}+\frac{|\xi|^{2}}{v} K_{1}\right](L-i) k, & L \geq i \geq 1 ; \\
\sum_{m=i}^{L-1}\left\|\omega^{m}-\omega^{m-1}\right\|^{2}+\sum_{m=i}^{L-1} \frac{1}{2} k\left\|A_{2} \omega^{m}\right\|^{2} & \\
\quad \leq K_{3}+\left[k\|g\|^{2}+C K_{2}\left(\frac{k}{\lambda_{2}}\|g\|^{2}+K_{1}\right)\right](L-i), & L \geq i \geq 1,
\end{array}
$$

where

$$
\begin{aligned}
& K_{2} \triangleq\left\|u^{0}\right\|\left\|^{2}+\frac{C}{v^{2} \lambda_{1}}\right\| f \|^{2}+\frac{N^{8} C}{v^{8} \lambda_{1}} K_{1}^{*}+\frac{C|\xi|^{2}}{v^{2} \lambda_{1}} K_{1}, \\
& K_{3} \triangleq \max \left\{\left\|\omega^{0}\right\|\left\|^{2}+\frac{\|g\|^{2}}{\frac{1}{2} \lambda_{2}-C K_{2}},\left[\frac{a_{3}}{k m_{1}}+a_{2}\right] e^{4 a_{1}}, C\right\| \omega^{0}\|\|^{2}+\frac{C}{K_{2}-\lambda_{2}}\|g\|^{2}\right\},
\end{aligned}
$$


$L \geq i \geq 1$

Proof. Taking the $L^{2}$ inner product of the equation (1.7) with $2 k A_{1} u^{m}$, we obtain

$$
\begin{aligned}
\left.\left\|u^{m}\right\|\right|^{2}+\left\|u^{m}-u^{m-1}\right\|^{2}+2 v k\left\|A_{1} u^{m}\right\|^{2} & \\
= & =\left\|u^{m-1}\right\|^{2}+2 k\left(f, A_{1} u^{m}\right)-2 k b_{N}\left(u^{m}, u^{m}, A_{1} u^{m}\right)-2 k\left(\xi \omega^{m}, A_{1} u^{m}\right) .
\end{aligned}
$$

Each term of the right hand side of the above equation can be majorize by (1.3) as follows

$$
\begin{aligned}
2 k\left(f, A_{1} u^{m}\right) & \leq 2 k\|f\|\left\|A_{1} u^{m}\right\| \leq \frac{k}{v}\|f\|^{2}+v k\left\|A_{1} u^{m}\right\|^{2} ; \\
2 k\left|\left(\xi \omega^{m}, A_{1} u^{m}\right)\right| & \leq \frac{16 k}{v}|\xi|^{2}\left\|\omega^{m}\right\|^{2}+\frac{v k}{16}\left\|A_{1} u^{m}\right\|^{2} ; \\
2 k\left|b_{N}\left(u^{m}, u^{m}, A_{1} u^{m}\right)\right| & =2 k F_{N}\left(\left\|u^{m}\right\|||\left|b\left(u^{m}, u^{m}, A_{1} u^{m}\right)\right|\right. \\
& \leq 2 k \frac{N}{\left\|u^{m}\right\|} C\left\|u^{m}\right\|^{\frac{1}{4}}\left\|A_{1} u^{m}\right\|^{\frac{3}{4}}\left\|u^{m}\right\|\left\|A_{1} u^{m}\right\| \\
& =C N k\left\|u^{m}\right\|^{\frac{1}{4}}\left\|A_{1} u^{m}\right\|^{\frac{7}{4}} \leq \frac{7}{8} v k\left\|A_{1} u^{m}\right\|^{2}+\frac{N^{8} C^{8} k}{8 v^{7}}\left\|u^{m}\right\|^{2} .
\end{aligned}
$$

Then

$$
\begin{aligned}
& \left\|u^{m}\right\|\left\|^{2}+\right\| u^{m}-u^{m-1}\left\|^{2}+2 v k\right\| A_{1} u^{m} \|^{2} \\
& \leq \mid\left\|u^{m-1}\right\|^{2}+\frac{k}{v}\|f\|^{2}+v k\left\|A_{1} u^{m}\right\|^{2}+\frac{7}{8} v k\left\|A_{1} u^{m}\right\|^{2} \\
& \quad+\frac{N^{8} C^{8} k}{8 v^{7}}\left\|u^{m}\right\|^{2}+\frac{16 k}{v}|\xi|^{2}\left\|\omega^{m}\right\|^{2}+\frac{v k}{16}\left\|A_{1} u^{m}\right\|^{2} .
\end{aligned}
$$

By (3.1) and (3.2) gives

$$
\begin{aligned}
& \left\|u^{m}\right\|^{2}+\left\|u^{m}-u^{m-1}\right\|^{2}+\frac{v k}{16}\left\|A_{1} u^{m}\right\|^{2} \\
& \leq\left\|u^{m-1}\right\|^{2}+\frac{k}{v}\|f\|^{2}+\frac{N^{8} C^{8} k}{8 v^{7}}\left\|u^{m}\right\|^{2}+\frac{16 k}{v}|\xi|^{2}\left\|\omega^{m}\right\|^{2} \\
& \leq\left\|u^{m-1}\right\|^{2}+\frac{k}{v}\|f\|^{2}+\frac{N^{8} C^{8} k}{8 v^{7}} K_{1}^{*}+\frac{16 k}{v}|\xi|^{2} K_{1},
\end{aligned}
$$

which together with $\left\|u^{m}\right\| \leq \frac{1}{\sqrt{\lambda_{1}}}\left\|A_{1} u^{m}\right\|$ gives

$$
\left\|u^{m}\left|\left\|^{2}+\right\| u^{m}-u^{m-1}\right|\right\|^{2}+\frac{v k \lambda_{1}}{16}\left\|u^{m}\right\|\left\|^{2} \leq\right\| u^{m-1}\left\|^{2}+\frac{k}{v}\right\| f \|^{2}+\frac{N^{8} C^{8} k}{8 v^{7}} K_{1}^{*}+\frac{16 k}{v}|\xi|^{2} K_{1} .
$$

Therefore

$$
\left[1+\frac{v k \lambda_{1}}{16}\right]\left\|u^{m}\right\|^{2} \leq\left\|\left.u^{m-1}\left|\left\|^{2}+\frac{k}{v}\right\| f \|^{2}+\frac{N^{8} C^{8} k}{8 v^{7}} K_{1}^{*}+\frac{16 k}{v}\right| \xi\right|^{2} K_{1},\right.
$$


which is

$$
\left\|u^{m} \mid\right\|^{2} \leq \frac{1}{1+\frac{v k \lambda_{1}}{16}}\left\|u^{m-1}\right\|^{2}+\frac{1}{1+\frac{v k \lambda_{1}}{16}}\left[\frac{k}{v}\|f\|^{2}+\frac{N^{8} C^{8} k}{8 v^{7}} K_{1}^{*}+\frac{16 k}{v}|\xi|^{2} K_{1}\right] .
$$

Using the above inequality recursively, we find

$$
\begin{aligned}
\left\|u^{m}\right\|^{2} \leq & \frac{1}{\left(1+\frac{v k \lambda_{1}}{16}\right)^{m}}\left\|u^{0}\right\|^{2}+\frac{16}{v k \lambda_{1}}\left[1-\frac{1}{\left(1+\frac{v k \lambda_{1}}{16}\right)^{m}}\right] \\
& \times\left[\frac{k}{v}\|f\|^{2}+\frac{N^{8} C^{8} k}{8 v^{7}} K_{1}^{*}+\frac{16 k}{v}|\xi|^{2} K_{1}\right] \\
\leq & \left\|u^{0}\right\|^{2}+\frac{16}{v k \lambda_{1}}\left[\frac{k}{v}\|f\|^{2}+\frac{N^{8} C^{8} k}{8 v^{7}} K_{1}^{*}+\frac{16 k}{v}|\xi|^{2} K_{1}\right] \\
\leq & \left\|u^{0}\right\|^{2}+\frac{C}{v^{2} \lambda_{1}}\|f\|^{2}+\frac{N^{8} C}{v^{8} \lambda_{1}} K_{1}^{*}+\frac{C|\xi|^{2}}{v^{2} \lambda_{1}} K_{1} \triangleq K_{2} .
\end{aligned}
$$

Therefore, we get (3.10), and $u^{m}$ is bounded in $\mathbb{V}$.

Taking the $L^{2}$ inner product of the equation (1.8) with $2 k A_{2} \omega^{m}$, we find

$$
\begin{aligned}
& \left\|\omega^{m}\right\|\left\|^{2}+\right\| \omega^{m}-\omega^{m-1}\|\|^{2}+2 k\left\|A_{2} \omega^{m}\right\|^{2} \\
& =\left\|\omega^{m-1}\right\|^{2}+2 k\left(g, A_{2} \omega^{m}\right)-2 k c\left(u^{m}, \omega^{m}, A_{2} \omega^{m}\right) \\
& \leq\left\|\omega^{m-1}\right\|^{2}+k\|g\|^{2}+k\left\|A_{2} \omega^{m}\right\|^{2}+C K_{2} k\left\|\omega^{m}\right\|^{2}+\frac{1}{2} k\left\|A_{2} \omega^{m}\right\|^{2} .
\end{aligned}
$$

Taking $k$ small enough such that $1-C K_{2} k>0$, we obtain

$$
\left(1-C K_{2} k\right)\left\|\omega^{m}\right\|\left\|^{2}+\frac{1}{2} k\right\| A_{2} \omega^{m}\left\|^{2}+\right\| \omega^{m}-\omega^{m-1}\|\|^{2} \leq\left.\left\|\omega^{m-1}\right\|\right|^{2}+k\|g\|^{2},
$$

which, together with $\left\|\omega^{m}\right\| \leq \frac{1}{\sqrt{\lambda_{2}}}\left\|A_{2} \omega^{m}\right\|$, leads to

$$
\left[1+\frac{1}{2} \lambda_{2} k-C K_{2} k\right]\left\|\omega^{m}\right\|^{2}+\left\|\omega^{m}-\omega^{m-1}\right\|^{2} \leq\left\|\omega^{m-1}\right\|^{2}+k\|g\|^{2} .
$$

There are two cases to discuss the above inequality (3.16):

Case 1: If $\frac{1}{2} \lambda_{2}>C K_{2}$, which is $1+\frac{1}{2} \lambda_{2} k-C K_{2} k>1$, then from (3.16), one has

$$
\left\|\omega^{m}\right\|^{2} \leq \frac{1}{1+\frac{1}{2} \lambda_{2} k-C K_{2} k}\left\|\omega^{m-1}\right\|^{2}+\frac{k}{1+\frac{1}{2} \lambda_{2} k-C K_{2} k}\|g\|^{2} .
$$

Using the above inequality recursively, we find

$$
\begin{aligned}
\left\|\omega^{m}\right\|^{2} \leq & \frac{1}{\left(1+\frac{1}{2} \lambda_{2} k-C K_{2} k\right)^{m}}\left\|\omega^{0}\right\|^{2} \\
& +\frac{1}{\frac{1}{2} \lambda_{2}-C K_{2}}\|g\|^{2}\left[1-\frac{1}{\left(1+\frac{1}{2} \lambda_{2} k-C K_{2} k\right)^{m}}\right]
\end{aligned}
$$




$$
\leq\left\|\omega^{0}\right\|^{2}+\frac{1}{\frac{1}{2} \lambda_{2}-C K_{2}}\|g\|^{2} .
$$

Case 2: If $\frac{1}{2} \lambda_{2} \leq C K_{2}$, which is $1+\frac{1}{2} \lambda_{2} k-C K_{2} k \leq 1$, then from (3.16), one gets

$$
\left[1-k\left(C K_{2}-\frac{1}{2} \lambda_{2}\right)\right]\left\|\omega^{m}\right\|^{2} \leq\left\|\omega^{m-1}\right\|\left\|^{2}+k\right\| g \|^{2} .
$$

In the following, we will use Lemma 3 to discuss the above inequality. Let $x_{m}=\left\|\omega^{m}\right\|^{2}, y_{m}=C K_{2}-\frac{1}{2} \lambda_{2}, z_{m}=\|g\|^{2}$. Obviously, $\left\{x_{m}\right\},\left\{y_{m}\right\},\left\{z_{m}\right\}$ are nonnegative sequences, and there exists $m_{0}, m_{1}$ such that

$$
\begin{aligned}
k y_{m} & =C K_{2} k-\frac{1}{2} k \lambda_{2}<\frac{1}{2}, & & \forall m \geq m_{0}, \\
\left(1-k y_{m}\right) x_{m} & \leq x_{m-1}+k z_{m}, & & \forall m>m_{0}+m_{1},
\end{aligned}
$$

and that for all integers $m * \geq m_{0}$, from (3.3), we get

$$
\begin{aligned}
& k \sum_{m=m *}^{m *+m_{1}} y_{m}=k \sum_{m=m *}^{m *+m_{1}}\left[C K_{2}-\frac{1}{2} \lambda_{2}\right]=k\left(m_{1}+1\right)\left[C K_{2}-\frac{1}{2} \lambda_{2}\right] \leq a_{1}, \\
& k \sum_{m=m *}^{m *+m_{1}} z_{m}=k \sum_{m=m *}^{m *+m_{1}}\|g\|^{2}=k\left(m_{1}+1\right)\|g\|^{2} \leq a_{2}, \\
& k \sum_{m=m *}^{m *+m_{1}} x_{m}=k \sum_{m=m *}^{m *+m_{1}}\left\|\omega^{m}\right\|^{2} \leq \frac{k}{\lambda_{2}}\|g\|^{2}\left(m_{1}+1\right)+K_{1} \leq a_{3} .
\end{aligned}
$$

By Lemma 3, we get

$$
\left\|\omega^{m}\right\|^{2} \leq\left[\frac{a_{3}}{k m_{1}}+a_{2}\right] e^{4 a_{1}}, \quad \forall m>m_{0}+m_{1} .
$$

When $m \leq m_{0}+m_{1}$, there is $\left[1+\frac{1}{2} \lambda_{2} k-C K_{2} k\right]^{m} \geq\left[1+\frac{1}{2} \lambda_{2} k-C K_{2} k\right]^{m_{0}+m_{1}}$, thus

$$
\begin{aligned}
\left\|\omega^{m}\right\|^{2} \leq & \frac{1}{\left(1+\frac{1}{2} \lambda_{2} k-C K_{2} k\right)^{m_{0}+m_{1}}}\left\|\omega^{0}\right\|^{2} \\
& +\frac{1}{C K_{2}-\frac{1}{2} \lambda_{2}}\|g\|^{2}\left[\frac{1}{\left(1+\frac{1}{2} \lambda_{2} k-C K_{2} k\right)^{m_{0}+m_{1}}}-1\right] \\
\leq & C\left\|\omega^{0}\right\|^{2}+\frac{C}{K_{2}-\lambda_{2}}\|g\|^{2}, \quad \forall m \leq m_{0}+m_{1} .
\end{aligned}
$$

Above all, we get (3.9), that is, $\omega^{m}$ is bounded in $H_{0}^{1}(\Omega)$.

Next, adding up (3.13) with $m=i, i+1, \cdots, L-1$, we find

$$
\left\|u^{L-1}\right\|\left\|^{2}+\sum_{m=i}^{L-1}\right\| u^{m}-u^{m-1} \mid\left\|^{2}+\sum_{m=i}^{L-1} \frac{v k}{16}\right\| A_{1} u^{m} \|^{2}
$$




$$
\leq\|\| u^{i-1} \|^{2}+(L-i)\left[\frac{k}{v}\|f\|^{2}+\frac{N^{8} C^{8} k}{8 v^{7}} K_{1}^{*}+\frac{16 k}{v}|\xi|^{2} K_{1}\right] .
$$

Together with (3.14), leads to (3.11). Using (3.5)-(3.6) and (3.15), we obtain

$$
\begin{aligned}
& \left\|\omega^{m}\right\|^{2}+\frac{1}{2} k\left\|A_{2} \omega^{m}\right\|^{2}+\left\|\omega^{m}-\omega^{m-1}\right\| \|^{2} \\
& \leq\left\|\omega^{m-1}\right\|^{2}+k\|g\|^{2}+C K_{2} k\left\|\omega^{m}\right\|^{2} \\
& \leq\left\|\omega^{m-1}\right\|^{2}+k\|g\|^{2}+C K_{2}\left[\frac{k}{\lambda_{2}}\|g\|^{2}+K_{1}\right] .
\end{aligned}
$$

Adding up the above inequality with $m=i, i+1, \cdots, L-1$, we get

$$
\begin{aligned}
&\left\|\omega^{L-1}\right\|^{2}+\sum_{m=i}^{L-1}\left\|\omega^{m}-\omega^{m-1}\right\|^{2}+\sum_{m=i}^{L-1} \frac{1}{2} k\left\|A_{2} \omega^{m}\right\|^{2} \\
& \leq\left\|\omega^{i-1}\right\|^{2}+(L-i)\left[k\|g\|^{2}+C K_{2}\left(\frac{k}{\lambda_{2}}\|g\|^{2}+K_{1}\right)\right] .
\end{aligned}
$$

Together with (3.9), one obtains (3.12). Above all, we have $\left\{u^{m}, \omega^{m}\right\}$ is bounded in $\mathbb{V} \times H_{0}^{1}(\Omega)$. The proof of Lemma 4 is completed.

3.3. Boundedness in $D\left(A_{1}\right) \times D\left(A_{2}\right)$

Lemma 5. Assuming that $f \in L^{2}(\Omega)^{3}, g \in L^{2}(\Omega), u_{0} \in D\left(A_{1}\right), \omega_{0} \in D\left(A_{2}\right)$. Let $\left\{u^{m}, \omega^{m}\right\}_{m \geq 1}$ be the solutions sequence of (1.7)-(1.8). Then there exist positive constants $C_{0} \equiv C_{0}\left(v, N,|\xi|, K_{1}, K_{2}, K_{3}, \quad\left\|A_{1} u^{0}\right\|,\left\|A_{2} \omega^{0}\right\|,\|f\|,\|g\|\right) \quad$ and $\quad K_{4} \equiv$ $K_{4}\left(k, \vee, \lambda_{1}, \lambda_{2}, N,|\xi|,\left\|u^{0}\right\|,\left\|\omega^{0}\right\|,\|f\|,\|g\|\right)$ such that

$$
\begin{gathered}
\left\|\frac{u^{1}-u^{0}}{k}\right\|+\left\|\frac{\omega^{1}-\omega^{0}}{k}\right\| \leq C_{0} ; \\
\left\|\frac{u^{m}-u^{m-1}}{k}\right\|^{2}+\left\|\frac{\omega^{m}-\omega^{m-1}}{k}\right\|^{2} \leq K_{4}, \quad m>1 .
\end{gathered}
$$

Proof. Let $u=u^{1}-u^{0}, \omega=\omega^{1}-\omega^{0}$, then from (1.7)-(1.8), one obtains

$$
\left\{\begin{array}{l}
\frac{u}{k}+v A_{1} u+v A_{1} u^{0}+B_{N}\left(u+u^{0}, u+u^{0}\right)+\xi\left(\omega+\omega^{0}\right)=f, \\
\frac{\omega}{k}+A_{2} \omega+A_{2} \omega^{0}+C\left(u+u^{0}, \omega+\omega^{0}\right)=g .
\end{array}\right.
$$

Taking the scalar product of the equation (3.19) with $A_{1} u$, we obtain

$$
\frac{\|u\|^{2}}{k}+v\left\|A_{1} u\right\|^{2}=\left(f-v A_{1} u^{0}, A_{1} u\right)-b_{N}\left(u+u^{0}, u+u^{0}, A_{1} u\right)-\left(\xi \omega+\xi \omega^{0}, A_{1} u\right) .
$$


Taking the scalar product of the equation (3.20) with $A_{2} \omega$, we find

$$
\frac{\|\omega\|^{2}}{k}+\left\|A_{2} \omega\right\|^{2}=\left(g-A_{2} \omega^{0}, A_{2} \omega\right)-c\left(u+u^{0}, \omega+\omega^{0}, A_{2} \omega\right) .
$$

Putting together (3.21) and (3.22), one obtains

$$
\begin{aligned}
& \frac{\|u\|^{2}}{k}+\frac{\|\omega\|^{2}}{k}+v\left\|A_{1} u\right\|^{2}+\left\|A_{2} \omega\right\|^{2} \\
& =\left(f-v A_{1} u^{0}, A_{1} u\right)+\left(g-A_{2} \omega^{0}, A_{2} \omega\right)-\left(\xi \omega+\xi \omega^{0}, A_{1} u\right) \\
& \quad-b_{N}\left(u+u^{0}, u+u^{0}, A_{1} u\right)-c\left(u+u^{0}, \omega+\omega^{0}, A_{2} \omega\right) .
\end{aligned}
$$

By (3.1) and (3.9)-(3.10) gives

$$
\begin{aligned}
& \left|b_{N}\left(u+u^{0}, u+u^{0}, A_{1} u\right)\right|=F_{N}\left(||\left|u+u^{0}\right|||\right)\left|b\left(u+u^{0}, u+u^{0}, A_{1} u\right)\right| \\
& \leq F_{N}\left(\left\|u+u^{0}\right\| \|\right)\left\|\left(u+u^{0}\right) \cdot \nabla\left(u+u^{0}\right)\right\|\left\|A_{1} u\right\| \\
& \leq F_{N}\left(\left\|u+u^{0}\right\|\right)\left\|u+u^{0}\right\|_{L_{6}}\left\|\nabla\left(u+u^{0}\right)\right\|_{L_{3}}\left\|A_{1} u\right\| \\
& \leq \frac{C N}{\left\|u+u^{0}\right\| \|}\left\|u+u^{0}\right\|\|\| u+u^{0}\left\|^{\frac{1}{2}}\right\| A_{1}\left(u+u^{0}\right)\left\|^{\frac{1}{2}}\right\| A_{1} u \| \\
& \leq C N\left\|u+u^{0}\right\|^{\frac{1}{2}}\left\|A_{1}\left(u+u^{0}\right)\right\|^{\frac{1}{2}}\left\|A_{1} u\right\| \\
& \leq C N K_{2}^{\frac{1}{4}}\left\|A_{1} u\right\|^{\frac{3}{2}}+C N K_{2}^{\frac{1}{4}}\left\|A_{1} u^{0}\right\|^{\frac{1}{2}}\left\|A_{1} u\right\| ; \\
& \left(f-v A_{1} u^{0}, A_{1} u\right) \leq\|f\|\left\|A_{1} u\right\|+v\left\|A_{1} u^{0}\right\|\left\|A_{1} u\right\| ; \\
& \left(g-A_{2} \omega^{0}, A_{2} \omega\right) \leq\|g\|\left\|A_{2} \omega\right\|+\left\|A_{2} \omega^{0}\right\|\left\|A_{2} \omega\right\| ; \\
& \left|c\left(u+u^{0}, \omega+\omega^{0}, A_{2} \omega\right)\right| \leq C\left|\left\|u ^ { 1 } \left|\left\|\left|\left\|\omega ^ { 1 } \left|\left\||| A_{2} \omega\right\| \leq C K_{2}^{\frac{1}{2}} K_{3}^{\frac{1}{2}}\left\|A_{2} \omega\right\| ;\right.\right.\right.\right.\right.\right.\right. \\
& \left|\left(\xi \omega+\xi \omega^{0}, A_{1} u\right)\right| \leq|\xi|\left\|\omega^{1}\right\|\left\|A_{1} u\right\| \leq|\xi| K_{1}^{\frac{1}{2}}\left\|A_{1} u\right\| .
\end{aligned}
$$

Therefore, one has

$$
\begin{aligned}
& \frac{\|u\| \|^{2}}{k}+\frac{\|\omega\| \|^{2}}{k}+v\left\|A_{1} u\right\|^{2}+\left\|A_{2} \omega\right\|^{2} \\
& \leq\|f\|\left\|A_{1} u\right\|+v\left\|A_{1} u^{0}\right\|\left\|A_{1} u\right\|+\|g\|\left\|A_{2} \omega\right\|+\left\|A_{2} \omega^{0}\right\|\left\|A_{2} \omega\right\| \\
& \quad+C N K_{2}^{\frac{1}{4}}\left\|A_{1} u\right\|^{\frac{3}{2}}+C N K_{2}^{\frac{1}{4}}\left\|A_{1} u^{0}\right\|^{\frac{1}{2}}\left\|A_{1} u\right\| \\
& \quad+C K_{2}^{\frac{1}{2}} K_{3}^{\frac{1}{2}}\left\|A_{2} \omega\right\|+|\xi| K_{1}^{\frac{1}{2}}\left\|A_{1} u\right\|,
\end{aligned}
$$

which, together with Young's inequality, leads to

$$
\begin{aligned}
& \frac{\|u\|^{2}}{k}+\frac{\|\omega\|^{2}}{k}+C\left\|A_{1} u\right\|^{2}+C\left\|A_{2} \omega\right\|^{2} \\
& \leq C\|f\|^{2}+C v\left\|A_{1} u^{0}\right\|^{2}+C\|g\|^{2}+C\left\|A_{2} \omega^{0}\right\|^{2}
\end{aligned}
$$




$$
+C N K_{2}+C N K_{2}^{\frac{1}{2}}\left\|A_{1} u^{0}\right\|+C K_{2} K_{3}+C|\xi|^{2} K_{1} .
$$

From (1.7)-(1.8), one has

$$
\left\{\begin{array}{l}
\frac{u^{1}-u^{0}}{k}=-v A_{1} u^{1}-B_{N}\left(u^{1}, u^{1}\right)-\xi \omega^{1}+f, \\
\frac{\omega^{1}-\omega^{0}}{k}=-A_{2} \omega^{1}-C\left(u^{1}, \omega^{1}\right)+g .
\end{array}\right.
$$

Thus

$$
\begin{aligned}
& \left\|\frac{u^{1}-u^{0}}{k}\right\|+\left\|\frac{\omega^{1}-\omega^{0}}{k}\right\| \\
& \leq v\left\|A_{1} u^{1}\right\|+\left\|A_{2} \omega^{1}\right\|+\left\|B_{N}\left(u^{1}, u^{1}\right)\right\|+\left\|C\left(u^{1}, \omega^{1}\right)\right\|+\left\|\xi \omega^{1}\right\|+\|f\|+\|g\| \\
& \leq v\left\|A_{1} u\right\|+v\left\|A_{1} u^{0}\right\|+\left\|A_{2} \omega\right\|+\left\|A_{2} \omega^{0}\right\| \\
& \quad+\left\|B_{N}\left(u^{1}, u^{1}\right)\right\|+\left\|C\left(u^{1}, \omega^{1}\right)\right\|+|\xi|\left\|\omega^{1}\right\|+\|f\|+\|g\| \\
& \leq v\left\|A_{1} u\right\|+v\left\|A_{1} u^{0}\right\|+\left\|A_{2} \omega\right\|+\left\|A_{2} \omega^{0}\right\| \\
& \quad+\left.C N\left\|u^{1}\right\|\right|^{\frac{1}{2}}\left\|A_{1} u^{1}\right\|^{\frac{1}{2}}+\left\|u^{1}\right\|\|\| \omega^{1}\||+\xi|\| \omega^{1}\|+\| f\|+\| g \| \\
& \leq v\left\|A_{1} u\right\|+v\left\|A_{1} u^{0}\right\|+\left\|A_{2} \omega\right\|+\left\|A_{2} \omega^{0}\right\|+C N K_{2}^{\frac{1}{4}}\left\|A_{1} u\right\|^{\frac{1}{2}} \\
& \quad+C N K_{2}^{\frac{1}{4}}\left\|A_{1} u^{0}\right\|^{\frac{1}{2}}+K_{2}^{\frac{1}{2}} K_{3}^{\frac{1}{2}}+|\xi| K_{1}^{\frac{1}{2}}+\|f\|+\|g\| .
\end{aligned}
$$

Which together with (3.23), gives the desired result, that is

$$
\left\|\frac{u^{1}-u^{0}}{k}\right\|+\left\|\frac{\omega^{1}-\omega^{0}}{k}\right\| \leq C_{0}\left(v, N,|\xi|, K_{1}, K_{2}, K_{3},\left\|A_{1} u^{0}\right\|,\left\|A_{2} \omega^{0}\right\|,\|f\|,\|g\|\right) .
$$

Then (3.17) is holds.

$$
\begin{aligned}
& \text { For } m>1 \text {, let } u_{*}^{m}=\frac{u^{m}-u^{m-1}}{k}, \omega_{*}^{m}=\frac{\omega^{m}-\omega^{m-1}}{k} \text { in (1.7)-(1.8), we obtain } \\
& \qquad \begin{array}{l}
\frac{u_{*}^{m}-u_{*}^{m-1}}{k}+v A_{1} u_{*}^{m}+\frac{1}{k}\left[B_{N}\left(u^{m}, u^{m}\right)-B_{N}\left(u^{m-1}, u^{m-1}\right)\right]+\xi \omega_{*}^{m}=0, \\
\frac{\omega_{*}^{m}-\omega_{*}^{m-1}}{k}+A_{2} \omega_{*}^{m}+\frac{1}{k}\left[C\left(u^{m}, \omega^{m}\right)-C\left(u^{m-1}, \omega^{m-1}\right)\right]=0 .
\end{array}
\end{aligned}
$$

Taking the scalar product of the equation (3.24) with $2 k u_{*}^{m}$, and taking the scalar product of the equation (3.25) with $2 k \omega_{*}^{m}$, we obtain

$$
\begin{aligned}
& \left\|u_{*}^{m}\right\|^{2}+\left\|u_{*}^{m}-u_{*}^{m-1}\right\|^{2}+2 k v\left\|u_{*}^{m}\right\|^{2}+\left\|\omega_{*}^{m}\right\|^{2}+\left\|\omega_{*}^{m}-\omega_{*}^{m-1}\right\|^{2}+2 k\left\|\omega_{*}^{m}\right\| \|^{2} \\
& =\left\|u_{*}^{m-1}\right\|^{2}-2 b_{N}\left(u^{m}, u^{m}, u_{*}^{m}\right)+2 b_{N}\left(u^{m-1}, u^{m-1}, u_{*}^{m}\right)-2 k\left(\xi \omega_{*}^{m}, u_{*}^{m}\right) \\
& \quad+\left\|\omega_{*}^{m-1}\right\|^{2}-2 c\left(u^{m}, \omega^{m}, \omega_{*}^{m}\right)+2 c\left(u^{m-1}, \omega^{m-1}, \omega_{*}^{m}\right) .
\end{aligned}
$$


We now majorize the right-hand side of (3.26). By (1.3) and (3.10), one gets

$$
\begin{aligned}
& 2 b_{N}\left(u^{m-1}, u^{m-1}, u_{*}^{m}\right)-2 b_{N}\left(u^{m}, u^{m}, u_{*}^{m}\right) \\
& =2 F_{N}\left(\left\|u^{m-1}\right\| \mid\right) b\left(u^{m-1}, u^{m-1}, u_{*}^{m}\right)-2 F_{N}\left(\left\|u^{m}\right\| \mid\right) b\left(u^{m}, u^{m}, u_{*}^{m}\right) \\
& =2\left[F_{N}\left(\left\|u^{m-1} \mid\right\|\right)-F_{N}\left(\left\|u^{m}\right\| \mid\right)\right] b\left(u^{m-1}, u^{m-1}, u_{*}^{m}\right) \\
& +2 F_{N}\left(\left\|u^{m}\right\|||\right)\left[b\left(u^{m-1}, u^{m-1}, u_{*}^{m}\right)-b\left(u^{m}, u^{m}, u_{*}^{m}\right)\right] \\
& \leq 2\left[F_{N}\left(\left\|u^{m-1}\right\| \mid\right)-F_{N}\left(\left\|u^{m}\right\|||\right)\right] b\left(u^{m-1}, u^{m-1}, u_{*}^{m}\right) \\
& +2 F_{N}\left(\||| u^{m}|| \mid\right)\left|k b\left(u_{*}^{m}, u^{m}, u_{*}^{m}\right)\right| \\
& \leq C \frac{\left\|u^{m}-u^{m-1}\right\| \mid}{\left\|u^{m-1} \mid\right\|}\left\|u^{m-1}\right\|\|\| u^{m-1}\|\mid\| u_{*}^{m}\left\|^{\frac{1}{4}}\right\| u_{*}^{m} \|^{\frac{3}{4}} \\
& +\frac{C N k}{\left\|u^{m}\right\|}\left\|u_{*}^{m}\right\|^{\frac{1}{4}}\left\|u_{*}^{m}\right\|^{\frac{3}{4}}\left\|u^{m}\right\|\|\| u_{*}^{m}\left\|^{\frac{1}{4}}\right\| u_{*}^{m} \|^{\frac{3}{4}} \\
& \leq C K_{2}^{\frac{1}{2}} k\left\|u_{*}^{m}\right\|^{\frac{1}{4}}\left\|u_{*}^{m}\right\|\left\|^{\frac{7}{4}}+C N k\right\| u_{*}^{m}\left\|^{\frac{1}{2}}\right\| u_{*}^{m} \|^{\frac{3}{2}}, \\
& -2 k\left(\xi \omega_{*}^{m}, u_{*}^{m}\right) \leq 2 k\left|\left(\xi \omega_{*}^{m}, u_{*}^{m}\right)\right| \leq 2 k|\xi|\left\|\omega_{*}^{m}\right\|\left\|u_{*}^{m}\right\| .
\end{aligned}
$$

Together with (3.9), one has

$$
\begin{aligned}
2 c\left(u^{m-1}, \omega^{m-1}, \omega_{*}^{m}\right)-2 c\left(u^{m}, \omega^{m}, \omega_{*}^{m}\right) & \leq 2\left|c\left(k u_{*}^{m}, \omega^{m}, \omega_{*}^{m}\right)\right| \\
& \leq C k\left\|u_{*}^{m}\right\|\|\| \omega^{m}\|\| \omega_{*}^{m}\left\|\leq C K_{3}^{\frac{1}{2}} k\right\| u_{*}^{m}\|\|\left\|\omega_{*}^{m}\right\| .
\end{aligned}
$$

Thus, from (3.26) and above inequality, by Young's inequality, we obtain

$$
\begin{aligned}
& \left\|u_{*}^{m}\right\|^{2}+\left\|u_{*}^{m}-u_{*}^{m-1}\right\|^{2}+2 k v\left\|u_{*}^{m}\right\|\left\|^{2}+\right\| \omega_{*}^{m}\left\|^{2}+\right\| \omega_{*}^{m}-\omega_{*}^{m-1}\left\|^{2}+2 k\right\| \omega_{*}^{m} \|^{2} \\
& \leq\left\|u_{*}^{m-1}\right\|^{2}+C K_{2}^{\frac{1}{2}} k\left\|u_{*}^{m}\right\| \frac{1}{4}\left\|u_{*}^{m}\right\|\left\|^{\frac{7}{4}}+C N k\right\| u_{*}^{m}\|\|^{\frac{1}{2}}\left\|u_{*}^{m}\right\| \|^{\frac{3}{2}} \\
& \quad+2 k|\xi|\left\|\omega_{*}^{m}\right\|\left\|u_{*}^{m}\right\|+\left\|\omega_{*}^{m-1}\right\|^{2}+C K_{3}^{\frac{1}{2}} k\left\|u_{*}^{m}\right\|\left\|\omega_{*}^{m}\right\| \\
& \leq\left\|u_{*}^{m-1}\right\|^{2}+\left\|\omega_{*}^{m-1}\right\|^{2}+C k\left\|u_{*}^{m}\right\|^{2}+2 k v\left\|u_{*}^{m}\right\|\left\|^{2}+C k\right\| \omega_{*}^{m} \|^{2} .
\end{aligned}
$$

Therefore

$$
(1-C k)\left(\left\|u_{*}^{m}\right\|^{2}+\left\|\omega_{*}^{m}\right\|^{2}\right) \leq\left\|u_{*}^{m-1}\right\|^{2}+\left\|\omega_{*}^{m-1}\right\|^{2} .
$$

Using the above inequality (3.27) recursively, we find

$$
\left\|u_{*}^{m}\right\|^{2}+\left\|\omega_{*}^{m}\right\|^{2} \leq \frac{1}{(1-C k)^{m-1}}\left(\left\|u_{*}^{1}\right\|^{2}+\left\|\omega_{*}^{1}\right\|^{2}\right) .
$$

Obviously, when $m \leq M_{0}=$ ent $\left\{\frac{T}{k}\right\}$, then $\left\|u_{*}^{m}\right\|^{2}+\left\|\omega_{*}^{m}\right\|^{2}$ is bounded, where ent $\left\{\frac{T}{k}\right\}$ is the entire part of $\frac{T}{k}$ with $T$ an arbitrarily fixed constant. Let $x_{m}=\left\|u_{*}^{m}\right\|^{2}+\left\|\omega_{*}^{m}\right\|^{2}$, $y_{m}=C, z_{m}=0$, then by Lemma 2-3 and equation (3.27), we see that $\left\|u_{*}^{m}\right\|^{2}+\left\|\omega_{*}^{m}\right\|^{2}$ 
is bounded as $m>M_{0}$. Thus we have

$$
\left\|\frac{u^{m}-u^{m-1}}{k}\right\|^{2}+\left\|\frac{\omega^{m}-\omega^{m-1}}{k}\right\|^{2} \leq K_{4}\left(v, \lambda_{1}, \lambda_{2}, N,|\xi|,\left\|u^{0}\right\|,\left\|\omega^{0}\right\|,\|f\|,\|g\|\right) .
$$

Then (3.18) is holds. The proof of Lemma 5 is completed.

Theorem 4. Assuming that $f \in L^{2}(\Omega)^{3}, g \in L^{2}(\Omega), u_{0} \in D\left(A_{1}\right), \omega_{0} \in D\left(A_{2}\right)$. Let $\left\{u^{m}, \omega^{m}\right\}_{m \geq 1}$ be the solution sequence of (1.7)-(1.8). Then there exists a positive constant $C$ such that, $\forall m \geq 1$,

$$
\left\|A_{1} u^{m}\right\|^{2}+\left\|A_{2} \omega^{m}\right\|^{2} \leq K_{5}
$$

Proof. From (1.7)-(1.8), we obtain

$$
\left\{\begin{array}{l}
v A_{1} u^{m}=-\frac{u^{m}-u^{m-1}}{k}-B_{N}\left(u^{m}, u^{m}\right)-\xi \omega^{m}+f, \\
A_{2} \omega^{m}=-\frac{\omega^{m}-\omega^{m-1}}{k}-C\left(u^{m}, \omega^{m}\right)+g .
\end{array}\right.
$$

Two sides of equation (3.30) and (3.31) multiply by $A_{1} u^{m}$ and $A_{2} \omega^{m}$ respectively, and then integrate gives

$$
\begin{aligned}
v & \left\|A_{1} u^{m}\right\|^{2}+\left\|A_{2} \omega^{m}\right\|^{2} \\
= & -\left[\frac{u^{m}-u^{m-1}}{k}, A_{1} u^{m}\right]-\left[\frac{\omega^{m}-\omega^{m-1}}{k}, A_{2} \omega^{m}\right]-b_{N}\left(u^{m}, u^{m}, A_{1} u^{m}\right) \\
& -c\left(u^{m}, \omega^{m}, A_{2} \omega^{m}\right)-\left(\xi \omega^{m}, A_{1} u^{m}\right)+\left(f, A_{1} u^{m}\right)+\left(g, A_{2} \omega^{m}\right) \\
\leq & \left\|\frac{u^{m}-u^{m-1}}{k}\right\|\left\|A_{1} u^{m}\right\|+\left\|\frac{\omega^{m}-\omega^{m-1}}{k}\right\|\left\|A_{2} \omega^{m}\right\| \\
& +\frac{C N}{\left\|u^{m}\right\|}\left\|\left(u^{m} \cdot \nabla\right) u^{m}\right\|\left\|A_{1} u^{m}\right\|+C\left\|u^{m}\right\|\|\| \omega^{m}\|\| A_{2} \omega^{m} \| \\
& +\left\|\xi \omega^{m}\right\|\left\|A_{1} u^{m}\right\|+\|f\|\left\|A_{1} u^{m}\right\|+\|g\|\left\|A_{2} \omega^{m}\right\| \\
\leq & \left\|\frac{u^{m}-u^{m-1}}{k}\right\|\left\|A_{1} u^{m}\right\|+\left\|\frac{\omega^{m}-\omega^{m-1}}{k}\right\|\left\|A_{2} \omega^{m}\right\|+C N\left\|u^{m}\right\|\left\|^{\frac{1}{2}}\right\| A_{1} u^{m} \|^{\frac{3}{2}} \\
& +C\left\|u^{m}\right\|\|\| \omega^{m}\|\| A_{2} \omega^{m}\|+\| \xi \omega^{m}\|\| A_{1} u^{m}\|+\| f\|\| A_{1} u^{m}\|+\| g\|\| A_{2} \omega^{m} \| .
\end{aligned}
$$

By Young's inequality, one gets

$$
\begin{aligned}
\left\|A_{1} u^{m}\right\|^{2}+\left\|A_{2} \omega^{m}\right\|^{2} \leq & C\left\|\frac{u^{m}-u^{m-1}}{k}\right\|^{2}+C\left\|\frac{\omega^{m}-\omega^{m-1}}{k}\right\|^{2}+C\left\|u^{m}\right\|^{2} \\
& +C\|\| u^{m}\|\|^{2}\left\|\omega^{m}\right\|^{2}+C|\xi|^{2}\left\|\omega^{m}\right\|^{2}+C\|f\|^{2}+C\|g\|^{2} \\
\leq & C K_{4}+C K_{2}+C K_{2} K_{3}+C|\xi|^{2} K_{1}+C\|f\|^{2}+C\|g\|^{2} \triangleq K_{5} .
\end{aligned}
$$

The proof of Theorem 4 is completed. 


\section{Global Attractor}

In this section, we first prove that continuous dependence of solutions on initial data and $N$.

Lemma 6. Assuming that $M, N>0, f \in L^{2}(\Omega)^{3}, g \in L^{2}(\Omega), u_{1}^{0}, u_{2}^{0} \in D\left(A_{1}\right)$, and $\omega_{1}^{0}, \omega_{2}^{0} \in D\left(A_{2}\right)$.

Let $\left\{u_{1}^{m}, \omega_{1}^{m}\right\}_{m}$ be the solution of (1.7)-(1.8), with initial condition $\left\{u_{1}^{0}, \omega_{1}^{0}\right\}$ and parameter $N$.

Let $\left\{u_{2}^{m}, \omega_{2}^{m}\right\}_{m}$ be the solution of (1.7)-(1.8), with initial condition $\left\{u_{2}^{0}, \omega_{2}^{0}\right\}$ and parameter $M$.

Then there exists $C, C_{*}, a_{1}^{*}, a_{2}^{*}$, $a_{3}^{*}$, such that

$$
\begin{array}{rlrl}
\left\|u_{1}^{m}-u_{2}^{m} \mid\right\|^{2}+\left\|\omega_{1}^{m}-\omega_{2}^{m}\right\|^{2} \leq & \frac{1}{C_{*}{ }^{M_{0}}}\left[\left.\left\|u_{1}^{0}-u_{2}^{0}\right\|\right|^{2}+\left\|\omega_{1}^{0}-\omega_{2}^{0}\right\| \|^{2}\right] \\
& +\frac{k C K_{5}}{1-C_{*}}\left[\frac{1}{C_{*}^{M_{0}}}-1\right]|M-N|^{2}, \quad \forall m \leq M_{0} ; \\
\left\|u_{1}^{m}-u_{2}^{m} \mid\right\|^{2}+\left\|\omega_{1}^{m}-\omega_{2}^{m}\right\|^{2} \leq & {\left[\frac{a_{3}^{*}}{k\left(M_{0}-3\right)}+a_{2}^{*}\right] e^{4 a_{1}^{*},} \quad} & \forall m>M_{0},
\end{array}
$$

where $M_{0}=$ ent $\left(\frac{T}{k}\right), T$ is an arbitrarily fixed constant.

Proof. Let $u_{*}^{m}=u_{1}^{m}-u_{2}^{m}, \omega_{*}^{m}=\omega_{1}^{m}-\omega_{2}^{m}$ in (1.7)-(1.8), we obtain

$$
\left\{\begin{array}{l}
\frac{u_{*}^{m}-u_{*}^{m-1}}{k}+v A_{1} u_{*}^{m}+B_{N}\left(u_{1}^{m}, u_{1}^{m}\right)-B_{M}\left(u_{2}^{m}, u_{2}^{m}\right)+\xi \omega_{*}^{m}=0, \\
\frac{\omega_{*}^{m}-\omega_{*}^{m-1}}{k}+A_{2} \omega_{*}^{m}+C\left(u_{1}^{m}, \omega_{1}^{m}\right)-C\left(u_{2}^{m}, \omega_{2}^{m}\right)=0 .
\end{array}\right.
$$

Taking the scalar product of (4.3) with $2 k A_{1} u_{*}^{m}$, we find

$$
\begin{aligned}
\| & \left.\left\|u_{*}^{m}\right\|\right|^{2}-\|\| u_{*}^{m-1}\|\|^{2}+\left\|u_{*}^{m}-u_{*}^{m-1}\right\|\left\|^{2}+2 v k\right\| A_{1} u_{*}^{m} \|^{2} \\
= & 2 k\left[b_{M}\left(u_{2}^{m}, u_{2}^{m}, A_{1} u_{*}^{m}\right)-b_{N}\left(u_{1}^{m}, u_{1}^{m}, A_{1} u_{*}^{m}\right)\right]-2 k\left(\xi \omega_{*}^{m}, A_{1} u_{*}^{m}\right) \\
= & 2 k F_{M}\left(\left\|\left|u_{2}^{m}\right|\right\|\right) b\left(u_{2}^{m}, u_{2}^{m}, A_{1} u_{*}^{m}\right) \\
& -2 k F_{N}\left(\| u_{1}^{m}||\right) b\left(u_{1}^{m}, u_{1}^{m}, A_{1} u_{*}^{m}\right)-2 k\left(\xi \omega_{*}^{m}, A_{1} u_{*}^{m}\right) \\
= & 2 k\left[F_{M}\left(\left\|\left|u_{2}^{m}\right|\right\|\right)-F_{N}\left(\left\|u_{1}^{m} \mid\right\|\right)\right] b\left(u_{1}^{m}, u_{2}^{m}, A_{1} u_{*}^{m}\right) \\
& -2 k F_{M}\left(\left\|u_{2}^{m} \mid\right\|\right) b\left(u_{*}^{m}, u_{2}^{m}, A_{1} u_{*}^{m}\right) \\
& -2 k F_{N}\left(\| u_{1}^{m}||\right) b\left(u_{1}^{m}, u_{*}^{m}, A_{1} u_{*}^{m}\right)-2 k\left(\xi \omega_{*}^{m}, A_{1} u_{*}^{m}\right) .
\end{aligned}
$$

From (1.3) and Lemma 1, one has

$$
F_{M}\left(\left\|u_{2}^{m} \mid\right\|\right)\left|b\left(u_{*}^{m}, u_{2}^{m}, A_{1} u_{*}^{m}\right)\right| \leq \frac{M}{\left\|u_{2}^{m}\right\| \mid} C\left\|u_{*}^{m} \mid\right\|^{\frac{1}{2}}\left\|u_{2}^{m}\right\|\|\| A_{1} u_{*}^{m}\left\|^{\frac{1}{2}}\right\| A_{1} u_{*}^{m} \|
$$




$$
\begin{aligned}
& =C M\left\|u_{*}^{m}\right\|\left\|^{\frac{1}{2}}\right\| A_{1} u_{*}^{m} \|^{\frac{3}{2}} ; \\
F_{N}\left(\left\|\left|u_{1}^{m}\right|\right\|\right)\left|b\left(u_{1}^{m}, u_{*}^{m}, A_{1} u_{*}^{m}\right)\right| & \leq\left|b\left(u_{1}^{m}, u_{*}^{m}, A_{1} u_{*}^{m}\right)\right| \\
& \leq C\left\|A_{1} u_{1}^{m} \mid\right\|\left\|u_{*}^{m}\right\|\left\|A_{1} u_{*}^{m}\right\| ;
\end{aligned}
$$

and

$$
\begin{aligned}
& {\left[F_{M}\left(\left\|\left|u_{2}^{m}\right|\right\|\right)-F_{N}\left(\left\|\left|u_{1}^{m}\right|\right\|\right)\right] b\left(u_{1}^{m}, u_{2}^{m}, A_{1} u_{*}^{m}\right)} \\
& \leq\left[\frac{|M-N|+\left|\left\|u_{1}^{m}-u_{2}^{m} \mid\right\|\right.}{\left\||| u_{2}^{m}\right\|}\right] C\left\|A_{1} u_{1}^{m}\right\|\|\| u_{2}^{m}\|\|\left\|A_{1} u_{*}^{m}\right\| \\
& \leq C\left[|M-N|+\left\|\left|u_{*}^{m}\right|\right\|\right]\left\|A_{1} u_{1}^{m}\right\|\left\|A_{1} u_{*}^{m}\right\| .
\end{aligned}
$$

From (4.5) and above inequality, we obtain

$$
\begin{aligned}
\| & \left\|u_{*}^{m}\right\|\left\|^{2}-\right\| u_{*}^{m-1}\left\|\left.\right|^{2}+\right\|\left|u_{*}^{m}-u_{*}^{m-1}\left\|\left.\right|^{2}+2 v k\right\| A_{1} u_{*}^{m} \|^{2}\right. \\
\leq & 2 k C\left[|M-N|+\left\|u_{*}^{m}\right\| \mid \|\right]\left\|A_{1} u_{1}^{m}\right\|\left\|A_{1} u_{*}^{m}\right\|+2 k C M\left\|u_{*}^{m}\right\|\left\|^{\frac{1}{2}}\right\| A_{1} u_{*}^{m} \|^{\frac{3}{2}} \\
& +2 k C\left\|A_{1} u_{1}^{m}\right\|\left\|\left|\left\|u_{*}^{m}\right\|\|\mid\| A_{1} u_{*}^{m}\|+2 k\| \xi \omega_{*}^{m}\|\| A_{1} u_{*}^{m} \|\right.\right. \\
\leq & {\left[\frac{k C|M-N|^{2}}{\varepsilon_{1}}+\frac{k C\left\|u_{*}^{m}\right\|^{2}}{\varepsilon_{2}}\right]\left\|A_{1} u_{1}^{m}\right\|^{2}+\left(k C \varepsilon_{1}+k C \varepsilon_{2}\right)\left\|A_{1} u_{*}^{m}\right\|^{2} } \\
& +\frac{k M^{4} C^{4}}{2 \varepsilon_{3}}\left\|u_{*}^{m}\right\|^{2}+\frac{3 k \varepsilon_{3}^{3}}{2}\left\|A_{1} u_{*}^{m}\right\|^{2}+\frac{k C^{2}}{\varepsilon_{4}}\left\|A_{1} u_{1}^{m}\right\|^{2}\left\|u_{*}^{m}\right\|^{2} \\
& +k \varepsilon_{4}\left\|A_{1} u_{*}^{m}\right\|^{2}+\frac{k|\xi|^{2}}{\varepsilon_{5} \lambda_{2}}\left\|\omega_{*}^{m}\right\|^{2}+k \varepsilon_{5}\left\|A_{1} u_{*}^{m}\right\|^{2} .
\end{aligned}
$$

Taking the scalar product of (4.4) with $2 k A_{2} \omega_{*}^{m}$, we find

$$
\begin{aligned}
\left\|\omega_{*}^{m}\left|\left\|^{2}-\right\| \omega_{*}^{m-1}\right|\right\|^{2}+\left\|\omega_{*}^{m}-\omega_{*}^{m-1}\right\|^{2}+2 k\left\|A_{2} \omega_{*}^{m}\right\|^{2} & =2 k\left[c\left(u_{2}^{m}, \omega_{2}^{m}, A_{2} \omega_{*}^{m}\right)-c\left(u_{1}^{m}, \omega_{1}^{m}, A_{2} \omega_{*}^{m}\right)\right] .
\end{aligned}
$$

By Young's inequality and (3.9)-(3.10) gives

$$
\begin{aligned}
& \left|c\left(u_{2}^{m}, \omega_{2}^{m}, A_{2} \omega_{*}^{m}\right)-c\left(u_{1}^{m}, \omega_{1}^{m}, A_{2} \omega_{*}^{m}\right)\right| \\
& =\left|c\left(u_{2}^{m}, \omega_{2}^{m}, A_{2} \omega_{*}^{m}\right)-c\left(u_{*}^{m}, \omega_{1}^{m}, A_{2} \omega_{*}^{m}\right)-c\left(u_{2}^{m}, \omega_{1}^{m}, A_{2} \omega_{*}^{m}\right)\right| \\
& =\left|c\left(u_{2}^{m}, \omega_{*}^{m}, A_{2} \omega_{*}^{m}\right)+c\left(u_{*}^{m}, \omega_{1}^{m}, A_{2} \omega_{*}^{m}\right)\right| \\
& \leq C|| u_{2}^{m}||||\left|\omega_{*}^{m}\right|||\left|A_{2} \omega_{*}^{m}\left\|+C|| u_{*}^{m}|||||| \omega_{1}^{m}|||| A_{2} \omega_{*}^{m}\right\|\right. \\
& \leq C\left[\frac{1}{2 \varepsilon_{6}}\left\|u_{2}^{m}\right\|\left\|^{2}\right\| \omega_{*}^{m} \mid\left\|^{2}+\frac{\varepsilon_{6}}{2}\right\| A_{2} \omega_{*}^{m} \|^{2}\right] \\
& +C\left[\frac{1}{2 \varepsilon_{7}}\left\|u_{*}^{m}\left|\left\|^{2}\right\| \omega_{1}^{m}\right|\right\|^{2}+\frac{\varepsilon_{7}}{2}\left\|A_{2} \omega_{*}^{m}\right\|^{2}\right] \\
& \leq C\left[\frac{1}{2 \varepsilon_{6}} K_{2}\left\|\omega_{*}^{m}\right\|^{2}+\frac{\varepsilon_{6}}{2}\left\|A_{2} \omega_{*}^{m}\right\|^{2}\right]
\end{aligned}
$$




$$
\begin{aligned}
& +C\left[\frac{1}{2 \varepsilon_{7}}\left\|u_{*}^{m}\right\|\left\|^{2} K_{3}+\frac{\varepsilon_{7}}{2}\right\| A_{2} \omega_{*}^{m} \|^{2}\right] \\
= & \frac{C K_{2}}{\varepsilon_{6}}\left\|\omega_{*}^{m}\right\|^{2}+\frac{C K_{3}}{\varepsilon_{7}}\left\|u_{*}^{m}\right\|^{2}+C\left(\varepsilon_{6}+\varepsilon_{7}\right)\left\|A_{2} \omega_{*}^{m}\right\|^{2} .
\end{aligned}
$$

Thus, from (4.7), we have

$$
\begin{aligned}
\left\|\omega_{*}^{m}\right\|\left\|^{2}-\right\| \omega_{*}^{m-1}\left\|\left.\right|^{2}+\right\| \omega_{*}^{m}-\omega_{*}^{m-1}\left\|^{2}+2 k\right\| A_{2} \omega_{*}^{m} \|^{2} \\
\quad \leq 2 k\left[\left.\frac{C K_{2}}{\varepsilon_{6}}\left\|\omega_{*}^{m}\right\|\right|^{2}+\left.\frac{C K_{3}}{\varepsilon_{7}}\left\|u_{*}^{m}\right\|\right|^{2}+C\left(\varepsilon_{6}+\varepsilon_{7}\right)\left\|A_{2} \omega_{*}^{m}\right\|^{2}\right] .
\end{aligned}
$$

From (4.6) and (4.8), we get

$$
\begin{aligned}
& \left.\left\|u_{*}^{m}\left|\left\|^{2}+\right\|\right| \omega_{*}^{m}\right\|\right|^{2}-\left\|\left|u_{*}^{m-1}\left\|\left.\right|^{2}-\right\| \omega_{*}^{m-1}\left\|\left.\right|^{2}+\right\| u_{*}^{m}-u_{*}^{m-1} \|^{2}\right.\right. \\
& +\left\|\omega_{*}^{m}-\omega_{*}^{m-1} \mid\right\|^{2}+2 v k\left\|A_{1} u_{*}^{m}\right\|^{2}+2 k\left\|A_{2} \omega_{*}^{m}\right\|^{2} \\
& \leq\left[\frac{k C|M-N|^{2}}{\varepsilon_{1}}+\frac{k C \mid\left\|u_{*}^{m}\right\|^{2}}{\varepsilon_{2}}\right]\left\|A_{1} u_{1}^{m}\right\|^{2}+\left(k C \varepsilon_{1}+k C \varepsilon_{2}\right)\left\|A_{1} u_{*}^{m}\right\|^{2} \\
& +\frac{k M^{4} C^{4}}{2 \varepsilon_{3}}\left\|u_{*}^{m} \mid\right\|^{2}+\frac{3 k \varepsilon_{3}^{3}}{2}\left\|A_{1} u_{*}^{m}\right\|^{2}+\frac{k C^{2}}{\varepsilon_{4}}\left\|A_{1} u_{1}^{m}\right\|^{2}\left\|u_{*}^{m}\right\| \|^{2} \\
& +k \varepsilon_{4}\left\|A_{1} u_{*}^{m}\right\|^{2}+\frac{k|\xi|^{2}}{\varepsilon_{5} \lambda_{2}}\left\|\omega_{*}^{m} \mid\right\|^{2}+k \varepsilon_{5}\left\|A_{1} u_{*}^{m}\right\|^{2} \\
& +2 k\left[\frac{C K_{2}}{\varepsilon_{6}}\left\|\omega_{*}^{m} \mid\right\|^{2}+\frac{C K_{3}}{\varepsilon_{7}}\left\|u_{*}^{m}\right\|\left\|^{2}+C\left(\varepsilon_{6}+\varepsilon_{7}\right)\right\| A_{2} \omega_{*}^{m} \|^{2}\right] \\
& \leq\left[\frac{k C|M-N|^{2}}{\varepsilon_{1}}+\frac{\left.k C\left\|u_{*}^{m}\right\|\right|^{2}}{\varepsilon_{2}}\right] K_{5}+\left(k C \varepsilon_{1}+k C \varepsilon_{2}\right)\left\|A_{1} u_{*}^{m}\right\|^{2} \\
& +\frac{k M^{4} C^{4}}{2 \varepsilon_{3}}\left\|u_{*}^{m} \mid\right\|^{2}+\frac{3 k \varepsilon_{3}^{3}}{2}\left\|A_{1} u_{*}^{m}\right\|^{2}+\frac{k C^{2}}{\varepsilon_{4}} K_{5}\left\|u_{*}^{m}\right\|^{2} \\
& +k \varepsilon_{4}\left\|A_{1} u_{*}^{m}\right\|^{2}+\frac{k|\xi|^{2}}{\varepsilon_{5} \lambda_{2}}\left\|\omega_{*}^{m} \mid\right\|^{2}+k \varepsilon_{5}\left\|A_{1} u_{*}^{m}\right\|^{2} \\
& +2 k\left[\frac{C K_{2}}{\varepsilon_{6}}\left\|\omega_{*}^{m}\right\|\left\|^{2}+\frac{C K_{3}}{\varepsilon_{7}}\right\| u_{*}^{m}\|\|^{2}+C\left(\varepsilon_{6}+\varepsilon_{7}\right)\left\|A_{2} \omega_{*}^{m}\right\|^{2}\right] \\
& =\left.\left\|u_{*}^{m}\right\|\right|^{2} k C\left[\frac{K_{5}}{\varepsilon_{2}}+\frac{M^{4}}{\varepsilon_{3}}+\frac{K_{5}}{\varepsilon_{4}}+\frac{K_{3}}{\varepsilon_{7}}\right]+\left\|A_{1} u_{*}^{m}\right\|^{2} k C\left(\varepsilon_{1}+\varepsilon_{2}+\varepsilon_{3}^{3}+\varepsilon_{4}+\varepsilon_{5}\right) \\
& +\left\|\left|\omega_{*}^{m}\right|\right\|^{2} k C\left[\frac{K_{2}}{\varepsilon_{6}}+\frac{|\xi|^{2}}{\varepsilon_{5} \lambda_{2}}\right]+\left\|A_{2} \omega_{*}^{m}\right\|^{2} k C\left(\varepsilon_{6}+\varepsilon_{7}\right)+\frac{k C K_{5}}{\varepsilon_{1}}|M-N|^{2} .
\end{aligned}
$$

That is

$$
\left[1-k C\left(K_{5}+M^{4}+K_{3}\right)\right]\left\|u_{*}^{m}\left|\left\|^{2}+\left[1-k C\left(K_{2}+\frac{|\xi|^{2}}{\lambda_{2}}\right)\right]\right\| \omega_{*}^{m}\right|\right\|^{2}
$$




$$
\begin{aligned}
& +\left\|u_{*}^{m}-u_{*}^{m-1}\left|\left\|^{2}+\right\|\right| \omega_{*}^{m}-\omega_{*}^{m-1}\right\|\left\|^{2}+k C\right\| A_{1} u_{*}^{m}\left\|^{2}+k C\right\| A_{2} \omega_{*}^{m} \|^{2} \\
\leq & \left.\left\|u_{*}^{m-1}\right\|\right|^{2}+\left\|\omega_{*}^{m-1}\right\|^{2}+k C K_{5}|M-N|^{2} .
\end{aligned}
$$

Let $C_{*}=\min \left\{1-k C\left(K_{5}+M^{4}+K_{3}\right), 1-k C\left(K_{2}+\frac{|\xi|^{2}}{\lambda_{2}}\right)\right\}$, then

$$
\left\|\left|u_{*}^{m}\right|\right\|^{2}+\left\|\left|\omega_{*}^{m}\right|\right\|^{2} \leq \frac{1}{C_{*}}\left[\left\|\left|u_{*}^{m-1}\right|\right\|^{2}+\|\left.\left|\omega_{*}^{m-1}\right|\right|^{2}\right]+\frac{k C K_{5}}{C_{*}}|M-N|^{2} .
$$

Using the above inequality recursively, we find

$$
\left\|\left.u_{*}^{m}\left|\left\|^{2}+\right\| \omega_{*}^{m}\right|\right|^{2} \leq \frac{1}{C_{*}^{m}}\left[\left\|u_{*}^{0}\right\|\left\|^{2}+\right\| \omega_{*}^{0} \|\left.\right|^{2}\right]+\frac{k C K_{5}}{1-C_{*}}|M-N|^{2}\left[\frac{1}{C_{*}^{m}}-1\right] .\right.
$$

Since $0<C_{*}<1$, for $m \leq M_{0}=$ ent $\left\{\frac{T}{k}\right\}$, one has

$$
\left\|\left|\left\|u_{*}^{m}\left|\left\|^{2}+\right\| \omega_{*}^{m}\right|\right\|^{2} \leq \frac{1}{C_{*}^{M_{0}}}\left[\left\|u_{*}^{0}\right\|\left\|^{2}+\right\|\left|\omega_{*}^{0} \|\right|^{2}\right]+\frac{k C K_{5}}{1-C_{*}}\right| M-\left.N\right|^{2}\left[\frac{1}{C_{*}^{M_{0}}}-1\right] .\right.
$$

For $m>M_{0}$, let $C_{* *}=\max \left\{C\left(K_{5}+M^{4}+K_{3}\right), C\left(K_{2}+\frac{|\xi|^{2}}{\lambda_{2}}\right)\right\}$. From (4.9), one has

$$
\left(1-k C_{* *}\right)\left|\left\|u_{*}^{m}||^{2}+\left(1-k C_{* *}\right)\right\|\right| \omega_{*}^{m}\left|\left\|^{2} \leq\right\|\right|\left|u_{*}^{m-1}\right|\left\|^{2}+\right\|\left|\omega_{*}^{m-1}\right| \|^{2}+k C K_{5}|M-N|^{2} .
$$

Let $x_{m}=\left\|u_{*}^{m}\left|\left\|^{2}+\right\|\right| \omega_{*}^{m}\left|\|^{2}, y_{m}=C_{* *}, z_{m}=C K_{5}\right| M-\left.N\right|^{2}\right.$. Obviously, $\left\{x_{m}\right\},\left\{y_{m}\right\}$, $\left\{z_{m}\right\}$ are non-negative sequences, and for $k>0$,

$$
\begin{aligned}
k y_{m}=k C_{* *} & <\frac{1}{2}, & \forall m \geq 2, \\
\left(1-k y_{m}\right) x_{m} & \leq x_{m-1}+k z_{m}, & \forall m \geq M-1 .
\end{aligned}
$$

For all integers $m_{*} \geq 2$, by Lemma 4 , we get

$$
\begin{aligned}
k \sum_{m=m_{*}}^{m_{*}+m_{1}} y_{m} & =k\left(M_{0}-2\right) C_{* *} \leq a_{1}^{*} ; \\
k \sum_{m=m_{*}}^{m m_{*}+m_{1}} z_{m} & =k\left(M_{0}-2\right) C K_{5}|M-N|^{2} \leq a_{2}^{*} ; \\
k \sum_{m=m_{*}}^{m_{*}+m_{1}} x_{m} & =k \sum_{m=m_{*}}^{m_{*}+m_{1}}\left(\left\|u_{1}^{m}-u_{2}^{m}\right\|^{2}+\left.\left\|\omega_{1}^{m}-\omega_{2}^{m}\right\|\right|^{2}\right) \\
& \leq k \sum_{m=m_{*}}^{m_{*}+m_{1}}\left(C\left|\left\|u_{1}^{m} \mid\right\|^{2}+C\left\|u_{2}^{m}\right\|^{2}+C\|\| \omega_{1}^{m}\left\|^{2}+C\right\| \omega_{2}^{m} \|^{2}\right)\right. \\
& \leq k C \sum_{m=m_{*}}^{m_{*}+m_{1}}\left(K_{2}+K_{2}+K_{3}+K_{3}\right) \leq k C\left(M_{0}-2\right)\left(K_{2}+K_{3}\right) \leq a_{3}^{*} .
\end{aligned}
$$


Thus, $x_{m} \leq\left[\frac{a_{3}^{*}}{k\left(M_{0}-3\right)}+a_{2}^{*}\right] e^{4 a_{1}^{*}}$, which is

$$
\left.\left\|u_{*}^{m}\right\|\right|^{2}+\left.\left\|\omega_{*}^{m}\right\|\right|^{2} \leq\left[\frac{a_{3}^{*}}{k\left(M_{0}-3\right)}+a_{2}^{*}\right] e^{4 a_{1}^{*}} .
$$

The proof of Lemma 6 is completed.

Proof of Theorem 2. Above, we show that the continuous dependence of solutions on initial value and parameter $N$. It can be seen under the above conditions, when determining the initial value and the parameter $N$, the system (1.7)-(1.8) has a unique solution. Therefore, we can define a $C^{0}$ semigroup $S^{m}$, acting on the phase space $\mathbb{V} \times H_{0}^{1}(\Omega)$, and defined as follows:

$$
S^{m}\left(u^{0}, \omega^{0}\right)=\left(u^{m}, \omega^{m}\right), \forall m \geq 0 .
$$

From Lemma 4, the semigroup $S^{m}$ has a bounded absorbing set in $\mathbb{V} \times H_{0}^{1}(\Omega)$ :

$$
B_{\mathbb{V} \times H_{0}^{1}(\Omega)}=\left\{\left(u^{m}, \omega^{m}\right) \in \mathbb{V} \times H_{0}^{1}(\Omega),\left\|u^{m}\right\|^{2}+\left\|\omega^{m}\right\|^{2} \leq K_{2}+K_{3}\right\} .
$$

And from Theorem 4 we can know that $S^{m}$ is bounded in $D\left(A_{1}\right) \times D\left(A_{2}\right)$, and using Sobolev embedding theorem to know that $S^{m}$ is compact in $\mathbb{V} \times H_{0}^{1}(\Omega)$. Hence, $S^{m}$ has a global attractor $\mathcal{A}$ in $\mathbb{V} \times H_{0}^{1}(\Omega)$. The proof of Theorem 2 is completed.

\section{LIMITING BEHAVIOR FOR $N \rightarrow \infty$}

One sees from Lemma 2 that

$$
v k\|\| u^{m} \mid\left\|^{2}+k\right\| \omega^{m}\left\|^{2} \leq \frac{2 k}{v \lambda_{1}}\right\| f\left\|^{2}+\frac{k}{\lambda_{2}}\right\| g \|^{2}+\left[1+\frac{2 k|\xi|^{2}}{v \lambda_{1}}\right] K_{1}+K_{1}^{*} .
$$

For $m$ and $k$ fixed, let $\left\{u_{N}^{m}, \omega_{N}^{m}\right\}_{N}$ be the solution of (1.7)-(1.8), then

$$
\left.\left\|u_{N}^{m}\right\|\right|^{2}+\left\|\omega_{N}^{m}\right\|^{2} \leq \frac{C}{v^{2} \lambda_{1}}\|f\|^{2}+\frac{C}{v \lambda_{2}}\|g\|^{2}+\frac{1}{v k}\left[\left(1+\frac{2 k|\xi|^{2}}{v \lambda_{1}}\right) K_{1}+K_{1}^{*}\right] .
$$

Thus the sequence $\left\{u_{N}^{m}, \omega_{N}^{m}\right\}_{N}$ is bounded in $\mathbb{V} \times H_{0}^{1}(\Omega)$ uniformly in $N$. Therefore, we can extract from $\left\{u_{N}^{m}, \omega_{N}^{m}\right\}_{N}$ a subsequence still denoted by $\left\{u_{N}^{m}, \omega_{N}^{m}\right\}_{N}$ such that $u_{N}^{m} \rightarrow u^{m}$, as $N \rightarrow \infty$ in $\mathbb{V}$, and $\omega_{N}^{m} \rightarrow \omega^{m}$, as $N \rightarrow \infty$ in $H_{0}^{1}(\Omega)$. As the injection $\mathbb{V} \hookrightarrow \mathbb{H}$ and $H_{0}^{1}(\Omega) \hookrightarrow L^{2}(\Omega)$ both are compact, we have $u_{N}^{m} \rightarrow u^{m}$, as $N \rightarrow \infty$ in $\mathbb{H}$, and $\omega_{N}^{m} \rightarrow \omega^{m}$ as $N \rightarrow \infty$ in $L^{2}(\Omega)$.

We shall show that

$$
\begin{cases}\lim _{N \rightarrow \infty} F_{N}\left(\left\|u_{N}^{m}\right\| \mid\right) b\left(u_{N}^{m}, u_{N}^{m}, v\right)=b\left(u^{m}, u^{m}, v\right), & \forall v \in D\left(A_{1}\right), \\ \lim _{N \rightarrow \infty} C\left(u_{N}^{m}, \omega_{N}^{m}, v_{*}\right)=c\left(u^{m}, \omega^{m}, v_{*}\right), & \forall v_{*} \in H^{2}(\Omega) .\end{cases}
$$

Indeed, a simple computation gives 


$$
\begin{aligned}
F_{N}\left(\left\|u_{N}^{m}\right\| \mid\right) b\left(u_{N}^{m}, u_{N}^{m}, v\right)-b\left(u^{m}, u^{m}, v\right) \\
=\left[F_{N}\left(\left\|u_{N}^{m}\right\|\right)-1\right] b\left(u_{N}^{m}, u_{N}^{m}, v\right)+b\left(u_{N}^{m}, u_{N}^{m}, v\right)-b\left(u^{m}, u^{m}, v\right) .
\end{aligned}
$$

First, by the definition of $F_{N}$, we have $F_{N}\left(\left\|u_{N}^{m}\right\|\right)=\min \left\{1, \frac{N}{\left\|u_{N}^{m}\right\|}\right\} \leq 1$. And from the above inequality we can see

$$
\frac{N}{\left\|u_{N}^{m}\right\|} \geq N\left\{\frac{C}{v^{2} \lambda_{1}}\|f\|^{2}+\frac{C}{v \lambda_{2}}\|g\|^{2}+\frac{1}{v k}\left[\left(1+\frac{2 k|\xi|^{2}}{v \lambda_{1}}\right) K_{1}+K_{1}^{*}\right]\right\}^{-\frac{1}{2}} .
$$

Hence, if $N>\left\{\frac{C}{v^{2} \lambda_{1}}\|f\|^{2}+\frac{C}{v \lambda_{2}}\|g\|^{2}+\frac{1}{v k}\left[\left(1+\frac{2 k|\xi|^{2}}{v \lambda_{1}}\right) K_{1}+K_{1}^{*}\right]\right\}^{\frac{1}{2}}$, we find that $F_{N}\left(|| u_{N}^{m} \mid \|\right)=1$. Therefore, $\lim _{N \rightarrow \infty} F_{N}\left(||\left|u_{N}^{m}\right| \|\right)=1$. Next, from (1.3), we obtain

$$
\begin{aligned}
b\left(u_{N}^{m}, u_{N}^{m}, v\right) & \leq C\left\|u_{N}^{m}\right\|\|\| u_{N}^{m}\|\|\left\|A_{1} v\right\| \\
& \leq C\left(\frac{C}{v^{2} \lambda_{1}}\|f\|^{2}+\frac{C}{v \lambda_{2}}\|g\|^{2}+\frac{1}{v k}\left[\left(1+\frac{2 k|\xi|^{2}}{v \lambda_{1}}\right) K_{1}+K_{1}^{*}\right]\right)\left\|A_{1} v\right\|,
\end{aligned}
$$

showing that $b\left(u_{N}^{m}, u_{N}^{m}, v\right)$ is bounded uniformly with respect to $N$, so

$$
\lim _{N \rightarrow \infty}\left[F_{N}\left(|| u_{N}^{m}|| \mid\right)-1\right] b\left(u_{N}^{m}, u_{N}^{m}, v\right)=0 .
$$

Using the strong convergence of $u_{N}^{m}$ in $\mathbb{H}$, we can prove as in [13], that $b\left(u_{N}^{m}, u_{N}^{m}, v\right) \rightarrow$ $b\left(u^{m}, u^{m}, v\right)$, as $N \rightarrow \infty$. Thus $\lim _{N \rightarrow \infty} F_{N}\left(\left\|u_{N}^{m}\right\| \mid\right) b\left(u_{N}^{m}, u_{N}^{m}, v\right)=b\left(u^{m}, u^{m}, v\right), \forall v \in D\left(A_{1}\right)$. Similarly, we have $\lim _{N \rightarrow \infty} c\left(u_{N}^{m}, \omega_{N}^{m}, v_{*}\right)=c\left(u^{m}, \omega^{m}, v_{*}\right), \forall v_{*} \in H^{2}(\Omega)$. Therefore, $\left\{u_{N}^{m}, \omega_{N}^{m}\right\}_{N}$ converges to the weak solution of the following equations when $N \rightarrow \infty$,

$$
\left\{\begin{array}{l}
\frac{u^{m}-u^{m-1}}{k}+v A_{1} u^{m}+B\left(u^{m}, u^{m}\right)+\xi \omega^{m}=f, \\
\frac{\omega^{m}-\omega^{m-1}}{k}+A_{2} \omega^{m}+C\left(u^{m}, \omega^{m}\right)=g .
\end{array}\right.
$$

Thus, we have completed the proof of Theorem 3.

\section{ACKNOWLEDGEMENT}

The author would like to thank the referee for the careful reading of this paper and for the valuable suggestions to improve the presentation and style of the paper.

\section{REFERENCES}

[1] A. M. Alghamdi, I. B. Omrane, S. Gala, and M. A. Ragusa, "A regularity criterion to the 3D Boussinesq equations," Sib. Electron. Math. Rep., vol. 16, no. 12, pp. 1795-1804, 2019, doi: 10.33048/semi.2019.16.127. 
[2] B. Birnir and N. Svanstedt, "Existence theory and strong attractors for the Rayleigh-Bénard problem with a large aspect ratio," Discr. Contin. Dynam. Syst. A, vol. 10, no. 1/2, pp. 53-74, 2012, doi: $10.3934 /$ dcds.2004.10.53.

[3] F. E. Browder, Existence and uniqueness theorems for solutions of nonlinear boundary value problems. New York: American Mathematical Society, Providence, 1965. doi: 10.1090/psapm/017/0197933.

[4] F. Cheng and C. J. Xu, "Analytical smoothing effect of solution for the Boussinesq equations," Acta Math. Sci., vol. 39B, no. 1, pp. 165-179, 2019, doi: 10.3969/j.issn.0252-9602.2019.01.014.

[5] G. Deugoue and J. K. Djoko, "On the time discretization for the globally modified three dimensional Navier-Stokes equations," J. Comput. Appl. Math., vol. 235, no. 8, pp. 2015-2029, 2010, doi: 10.1016/j.cam.2010.10.003.

[6] S. Gala and M. A. Ragusa, "A regularity criterion of weak solutions to the 3D Boussinesq equations," Bullet. Brazilian Math. Society, vol. 50, no. 3, 2019, doi: 10.1007/s00574-019-00162-z.

[7] A. Huang and W. Huo, "The global attractor of the 2D Boussinesq equations with fractional Laplacian in Subcritical case," Discr. Contin. Dynam. Syst. B, vol. 21, no. 8, pp. 2531-2550, 2017, doi: $10.3934 /$ dcdsb.2016059.

[8] A. V. Kapustyan, A. V. Pankov, and J. Valero, "On Global Attractors of Multivalued Semiflows Generated by the 3D Bénard System,” Set-Valued Variat. Analy., vol. 20, no. 4, pp. 445-465, 2012, doi: 10.1007/s11228-011-0197-5.

[9] O. V. Kapustyan, V. S. Melnik, and J. Valero, "A weak attractor and properties of solutions for the three-dimensional Bénard problem,” Discr. Contin. Dynam. Syst., vol. 18, no. 2/3, pp. 449-481, 2007, doi: 10.3934/dcds.2007.18.449.

[10] O. V. Kapustyan and A. V. Pankov, "Global $\varphi$-attractor for a modified 3D Bénard system on channel-like domains," Nonauto. Stochastic Dyna. Syst., vol. 1, no. 1, pp. 1-9, 2014, doi: 10.2478/msds-2013-0001.

[11] O. V. Kapustyan, A. V. Pankov, and J. Valero, "On the existence and connectedness of a global attractor for solutions of the three-dimensional Bénard system that satisfy a system of energy inequalities," J. Math. Sci., vol. 191, no. 3, pp. 388-402, 2013, doi: 10.1007/s10958-013-1325-6.

[12] M. Kaya and A. O. Çelebi, "Global attractor for the regularized Bénard problem," Appl. Analy., vol. 93, no. 9, pp. 1989-2001, 2014, doi: 10.1080/00036811.2013.867018.

[13] R. Temam, Theory and Numerical Analysis of the Navier-Stokes Equations. New York: North Holland Publishing company, 1977.

[14] X. M. Wang, "Asymptotic Behavior of the Global Attractors to the Boussinesq System for Rayleigh-Bénard Convection at Large Prandtl Number," Commun. Pure Appli. Math., vol. 60, no. 9, pp. 1293-1318, 2007, doi: 10.1002/cpa.20170.

[15] C. S. Zhu, "Attractor of a semi-discrete Benjamin-Bona-Mahony equation on $\mathbb{R}^{1}$," Annales Polonici Math., vol. 115, no. 3, pp. 219-234, 2015, doi: 10.4064/ap115-3-2.

[16] Z. Q. Zhu, "Stable analysis for a delayed population model with survival rate," Miskolc Math. Notes, vol. 20, no. 1, pp. 611-624, 2019, doi: 10.18514/MMN.2019.2675.

Author's address

Chaosheng Zhu

Southwest University, School of Mathematics and Statistics, Chongqing, 400715, P. R. China

E-mail address: zcs@swu.edu.cn 